\title{
Dinámica del fitoplancton en un embalse de alta cota del Noroeste Argentino (Tucumán)
}

\author{
Claudia Seeligmann ${ }^{1, *}$ y Beatriz C. Tracanna ${ }^{1,2,3}$ \\ ${ }^{1}$ Facultad de Ciencias Naturales e Instituto Miguel Lillo, Universidad Nacional de Tucumán, Miguel Lillo 205, \\ 4000 San Miguel de Tucumán, Argentina. \\ ${ }^{2}$ Fundación Miguel Lillo. \\ ${ }^{3}$ Consejo de Investigaciones Científicas y Técnicas.
}

* Autor responsable de la correspondencia: cseeligmann@ hotmail.com

Recibido: 26/11/07

Aceptado: 15/1/09

\begin{abstract}
Phytoplankton dynamics in a high elevation reservoir of Northwestern Argentina (Tucuman)

In 2000 limnological studies were conducted in La Angostura Dam and at the estuaries of its tributaries, at $1980 \mathrm{~m}$ a.s.1. in Tucumán province (Argentina). In this paper the results about the physical and chemical characteristics of the dam, the trophic state and the composition and dynamics of the phytoplankton community, related to the abiotic variables are presented. Due to the thermal behavior of the dam, La Angostura would classify as a warm monomictic type and the water was characterized as: $\mathrm{HCO}_{3}^{-}>\mathrm{SO}_{4}^{=}>\mathrm{Cl}^{-} ; \mathrm{Ca}^{2+}>\mathrm{Na}^{+}>\mathrm{K}^{+}>\mathrm{Mg}^{2+}$. A total of 66 algae taxa were recorded, with an average abundance ranging from $179 \mathrm{ind} / \mathrm{ml}$ to $2.122 \mathrm{ind} / \mathrm{ml}$. Because it is subtropical lake located in a mountain area, La Angostura did not correspond with a defined trophic category. Due to a decrease in diversity at higher abundance, high $\mathrm{pH}$, high phytoplanktonic biomass fluctuations, and readings lower than 0.005 and $0.5 \mathrm{mg} / \mathrm{l}$ for nitrite and nitrate respectively, the dam would classify as eutrophic; and due to the $\alpha$ chlorophyll, dissolved oxygen, oxygen biochemical demand, and ammonium, it would classify as an oligo-mesotrophic type body of water. The results obtained from the multivariate analysis allowed to confirm that the phytoplankton composition was firstly determined by the temperature, which controlled the physical mixture of the water column, and which additionally was affected by strong winds. Secondly, the phytoplankton composition depended upon the hydrological cycle, as this was the parameter that was most influential on the water transparency. Lastly, the different trophic indicators also affected on the phyto|plankton dynamic.
\end{abstract}

Key words: Phytoplankton, physical-chemical variables, trophic level, mountain dam.

\section{RESUMEN}

\section{Dinámica del fitoplancton en un embalse de alta cota del Noroeste Argentino (Tucumán)}

En el año 2000 se realizaron estudios limnológicos en el embalse La Angostura y en la desembocadura de sus tributarios, situado a 1980 msnm, en la provincia de Tucumán (Argentina). En este trabajo se dan a conocer los resultados sobre las características físicas y químicas del embalse, estado trófico, y sobre la composición y dinámica de la comunidad fitoplanctónica, en relación con las variables abióticas. Por el comportamiento térmico del embalse La Angostura estaría dentro del tipo monomíctico cálido y las aguas se caracterizaron como: $\mathrm{HCO}_{3}^{-}>\mathrm{SO}_{4}^{=}>\mathrm{Cl}^{-} ; \mathrm{Ca}^{2+}>\mathrm{Na}^{+}>\mathrm{K}^{+}>\mathrm{Mg}^{2+}$. Se registraron un total de 66 taxones algales, con una abundancia promedio entre $179 \mathrm{ind} / \mathrm{ml} \mathrm{a} 2122 \mathrm{ind} / \mathrm{ml}$. Por tratarse de un lago subtropical pero emplazado en una zona montañosa, La Angostura no se ajustó a una categoría trófica definida: por la disminución de la diversidad a mayor abundancia, elevado $\mathrm{pH}$, grandes fluctuaciones de la biomasa fitoplanctónica, y registros menores a 0.005 y $0.5 \mathrm{mg} / \mathrm{l}$ de nitrito y nitrato respectivamente, se lo podría clasificar como eutrófico y por los niveles de clorofila a, oxígeno disuelto, demanda bioquímica de oxígeno y amonio lo ubicarían dentro de un cuerpo de agua del tipo oligo-mesotrófico. Los resultados obtenidos a partir de los análisis multivariados permitieron confirmar que la composición del fitoplancton estuvo en primer lugar determinada por la temperatura, la que ejerció control sobre mezcla física de la columna de agua, la que se vio afectada, como factor adicional, por fuertes vientos. En segundo lugar, dependió del ciclo hidrológico ya que fue el parámetro que más influyó sobre la transparencia del agua. Por último, los distintos indicadores tróficos también intervinieron en la dinámica del fitoplancton.

Palabras clave: Fitoplancton, variables físico-químicas, estado trófico, embalse de montaña. 


\section{INTRODUCCIÓN}

Los estudios efectuados en las últimas décadas en los ecosistemas acuáticos, demostraron que éstos sufren profundas modificaciones como producto de las actividades antrópicas y una de las causas de este fenómeno se debe al aumento en la generación de residuos sólidos y líquidos, situación que ha avanzado de modo considerable y globalmente (Dolbeth et al., 2003; Fontúrbel, 2004; Western, 2001). Esto trae aparejado un incremento en la concentración de nutrientes que va más allá de los límites admisibles, ocasionando una desestabilización y degradación de los ecosistemas, cuya incidencia más directa es el deterioro de la calidad de agua, que en algunos casos es irreversible y en otros produce significativas pérdidas económicas (Carpenter \& Cottingham, 1997). Por otra parte la eutrofización, ocasiona un incremento y una alteración en la composición del fitoplancton (Dolbeth et al., 2003; Höfle et al., 1999; Reynolds, 1998; Western, 2001). Numerosos autores (Seip \& Reynolds, 1995; Rojo, 1998; del Giorgio et. al., 1991; Rott, 1998) concuerdan que la abundancia de especies fitoplanctónicas y la estructura de esta comunidad varían a lo largo de gradientes tróficos y estacionales. Bajo este aspecto creció el interés en el manejo de lagos y embalses, usando asociaciones de especies como indicadoras de niveles tróficos (Huszar et al., 1998). Sin embargo, la alteración en la composición de especies no siempre es directa respecto a la disponibilidad de nutrientes, ya que otros factores se encuentran involucrados (Reynolds, 1998). La utilización de modelos de calidad del agua, específicamente en lagos, ha aumentado notoriamente, sobre todo en zonas templadas. A pesar de la idea que esta metodología debe ser estándar y aplicable a los lagos del mundo, son necesarias más investigaciones en las zonas tropicales y subtropicales, donde se han realizado muy pocos estudios de este tipo, para poder comprobar si la misma se puede realmente generalizar. Por esto, si bien, los estudios ecológicos del fitoplancton en ambientes acuáticos tropicales son escasos y recientes comparados con los producidos para los lagos del hemisferio norte (Zalocar et al., 1998), se conoce que las causas que condicionan la distribución de especies son numerosas, entre las que se incluyen altas temperaturas, diferencias en la estabilidad térmica y circulación de nutrientes (Huszar et al., 1998). Para otros autores, además del nivel trófico, consideran que un importante regulador de la composición del fitoplancton estaría dado por el grado de dureza del agua (Dasí et al., 1998). Este factor está mas relacionado con la geología y el clima del lugar, mientras que el nivel trófico con influencias antropogénicas.

Otro tipo de contaminación particularmente importante en los sistemas hídricos, es la provocada por metales pesados, la que produce efectos adversos en las distintas comunidades acuáticas (Topalián et al., 1999). Estos componentes provienen ya sea de las actividades mineras o de las agrícolas. A bajas concentraciones, muchos de los metales juegan un papel esencial en el metabolismo de las microalgas, tal es el caso del Zn y $\mathrm{Cu}$. Sin embargo, cuando las concentraciones aumentan de manera excesiva provocan desbalances metabólicos en el fitoplancton (Rodríguez \& Rivera, 1995). Un ejemplo de ello es la inhibición del crecimiento, así como cambios morfológicos, generados como una respuesta fisiológica a la exposición del metal. Rodríguez y Rivera (1995) afirman que el cobre actúa como un agente algistático para Tetraselmis suecica y Dunaliella salina entre concentraciones de 5.0 a $10.0 \mathrm{mg} / \mathrm{l}$ de sulfato de cobre.

Por último, con el fin de almacenar agua para distintos usos, las cuencas hídricas sufren grandes alteraciones debido a la construcción de embalses, la que modifica simultáneamente los cursos naturales, el régimen de caudales, la estructura física del hábitat y el funcionamiento ecológico (Prenda et al., 2006).

La dinámica de lagos y embalses de alta cota se diferencian considerablemente de aquéllos que se encuentran en altitudes bajas debido a las condiciones climáticas. Los lagos tropicales de alta montaña son ecosistemas acuáticos particulares y se conoce muy poco sobre los mismos (Gunkel, 2003). Investigaciones han mostrado que en general estos ambientes son clasificados como oligotérmicos (nunca se estratifican) y de darse una estratificación, ésta es poco estable y de corta dura- 
ción. Se caracterizan también por un aislamiento geográfico, poseen temperaturas medias por debajo de $20^{\circ} \mathrm{C}$ y valores de saturación de oxígeno bajos (Casallas y Gunkel, 2001). Por otro lado, los lagos de montaña son particularmente más sensibles a los cambios ambientales por las condiciones climáticas extremas, lo que desarrolla ecosistemas lábiles y simples, que reaccionan rápidamente al estrés ambiental (Nauwerck, 1994).

Los estudios sobre ecosistemas acuáticos artificiales para la provincia de Tucumán se centraron principalmente en zonas entre 200$650 \mathrm{~m}$ snm (Tracanna, et al., 1992; Tracanna \& Seeligmann, 1993; Seeligmann \& Tracanna, 1994; Tracanna et al., 1994; Tracanna et al., 1996; Locascio de Mitrovich et al., 1997; Tracanna et al., 1999, entre otros). Esta es la primera contribución limnológica realizada en un embalse de alta cota en la provincia.

En este estudio aportamos información sobre las características físicas y químicas del embalse, estado trófico y sobre la composición y dinámica de la comunidad fitoplanctónica y la desembocadura de sus tributarios, en relación a las variables abióticas.

\section{ÁREA DE ESTUDIO}

El embalse La Angostura, localizado aproximadamente a $100 \mathrm{~km}$ al sudoeste de la ciudad de San Miguel de Tucumán (Argentina) (265' S y $65^{\circ} 41^{\prime} \mathrm{W}$ ), está ubicado en una zona montañosa del Departamento Tafí del Valle, a los 1980 m snm (Fig. 1). La Cuenca del río La Angostura es una depresión tectónica hundida por fallas principales de rumbo NNW-SSE y cortada transversalmente en su extremo Sur por fallas secundarias E-W. El rellenado de la depresión está conformado por tres tipos de material: limos loessicos, aluviones de arenas y gravas (Mochkopsky, 2002). El espejo del agua tiene forma aproximadamente triangular con un largo máximo de $4 \mathrm{~km}$ y un ancho de $2.5 \mathrm{~km}$, una profundidad máxima de $40 \mathrm{~m}$, un volumen de $833000 \mathrm{~m}^{3}$ y un tiempo de residencia de 0.1 años. Las precipitaciones anuales fluctúan entre $200-400 \mathrm{~mm}$, concentradas especialmente durante el verano. La temperatura ambiente varía entre los $8-26{ }^{\circ} \mathrm{C}$. Los tributarios na- cen en las cumbres Calchaquíes y reciben los residuos de las actividades agrícolas, ganaderas y urbanas de la zona, con lo cual se ha incrementado el riesgo hacia una eutrofización.

El embalse se encuentra en servicio desde el año 1978 y fue construido para atenuación de crecidas, agua para consumo, turismo, industrias y diversificación agropecuaria y energía hidroeléctrica; no habiéndose explotado este último aprovechamiento hasta el presente (Mochkopsky, loc. cit.; Zavala de Chokoff, 2002). La presa se encuentra próxima a dos importantes villas veraniegas y desembocan en ésta los ríos: Tafí y El Mollar. En el Valle de Tafí en los últimos años se incrementó la población y la actividad agrícola ganadera, produciendo alteraciones antrópicas por falta de programas de manejo (Isasmendi et al., 2007). Estos autores detectaron en los ríos de la zona, bajos tenores en los parámetros indicadores de eutrofización (fosfato, nitrato, $\mathrm{DBO}_{5}$ ), en general un $\mathrm{pH}$ alcalino y pudieron registrar la presencia de cobre y hierro. Los ríos de las áreas montañosas en la provincia de Tucumán están sujetos a los regímenes estacionales extremos que se caracterizan por la alternancia de períodos extremadamente secos durante el invierno y flujos casi catastróficos en el verano por el aumento de caudal, por lo que las evacuaciones del agua se realizan de acuerdo a las necesidades para evitar desbordes durante el verano.

\section{MATERIAL Y MÉTODOS}

A partir de diciembre de 2000 y durante un año, se efectuaron muestreos bimensuales en tres puntos: en la zona más profunda del embalse (zona limnética) y en las desembocaduras de los tributarios (Fig. 1). En la primera se tomaron muestras a distintas profundidades (superficie, nivel de la zona fótica, 8 metros y fondo) para oxígeno disuelto (OD), clorofila $a$ y cuali-cuantitativas del fitoplancton y superficiales, para análisis de iones mayoritarios, compuestos nitrogenados y ortofosfato. En esta zona se midió "in situ", perfiles de temperatura, conductividad y $\mathrm{pH}$ con equipo digital marca Altronix. En las desembocaduras de los ríos las mediciones y extracciones 
de muestras se realizaron sólo en superficie. En los tres sitios muestreados se estimó la transparencia, mediante el disco de Secchi, la concentración de metales pesados ( $\mathrm{Mn}, \mathrm{Cu}, \mathrm{As}, \mathrm{Fe})$ y la demanda bioquímica de oxígeno $\left(\mathrm{DBO}_{5}\right)$. $\mathrm{La}$ profundidad de la zona eufótica $\left(Z_{\text {eu }}\right)$ fue calculada de acuerdo a Bormans et al., 2005, como así también la relación $\mathrm{Z}_{\mathrm{m}} / \mathrm{Z}_{\mathrm{eu}}$. Las muestras para el análisis de OD se fijaron en el campo y se determinaron en laboratorio por medio del

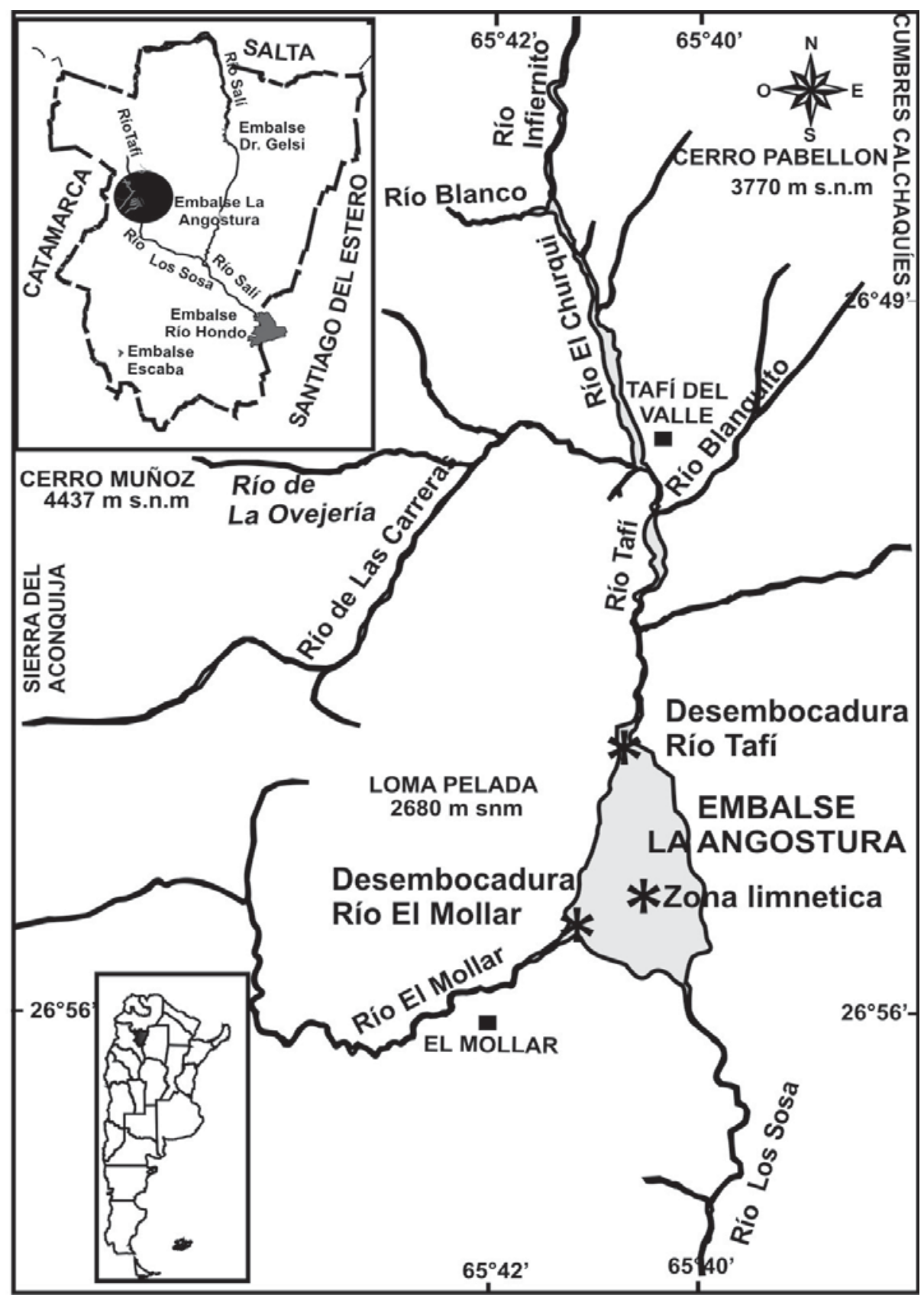

Figura 1. Lugares de muestreo: zona limnética del embalse La Angostura y desembocaduras de los ríos Mollar y Tafí. Sampling sites: Limnetic zone of La Angostura dam and estuaries of the El Mollar and Tafi Rivers. 
método de Winkler modificado (APHA, 1992), mientras que para los análisis químicos de iones mayoritarios, $\mathrm{DBO}_{5}$, compuestos nitrogenados, ortofosfato, y metales pesados se extrajeron en recipientes plásticos de 1.5 litros, lavados previamente con agua destilada y llevados a laboratorio. Para el análisis de estos últimos se utilizó ácido nítrico diluido en una proporción 3:1 y se fijaron en campo con 5 gotas de ácido nítrico concentrado. Todas las muestras fueron trasladadas al laboratorio en frío y oscuridad. La metodología que se siguió para los análisis químicos fue la recomendada en APHA (1992).

Las muestras algales cualitativas fueron tomadas con red de plancton de $25 \mu \mathrm{m}$ de abertura de malla y las cuantitativas con botellas de Ruttner de $500 \mathrm{ml}$ de capacidad, ambas fijadas con formaldehído al $4 \%$.

Para el análisis de bacterias (totales, fecales y Streptofecales) se extrajeron muestras en envases estériles, en distintos niveles de la zona limnética y en superficie de las desembocaduras de los ríos. Los parámetros físicos, químicos y bacteriológicos se analizaron siguiendo la metodología standard (APHA, 1992). La clorofila $a$ (extracción de muestras, procesamiento y cálculo), fue evaluada siguiendo a Loez (1995). Los análisis cuantitativos del fitoplancton se realizaron según la metodología de Utermöhl (1958) y la diversidad específica se calculó de acuerdo a Shannon \& Weaver (1963).

Los análisis de componentes principales (ACP-1, ACP-2 y ACP-3) se efectuaron usando Statistica 6.0 (StatSoft, 1984-2001, Tulsa, OK, USA). Para el ACP-1 se seleccionaron 17 especies fitoplanctónicas, en base a una frecuencia de ocurrencia mayor al $50 \%$, usando transformación logarítmica de las densidades. El ACP-2 fue realizado con los dos primeros componentes del ACP-1 y las variables abióticas transformadas logarítmicamente (temperatura, transparencia, $\mathrm{pH}$, conductividad, OD y DBO 5 ) El ACP-3 (no mostrado) se efectúo sólo con los parámetros físicos y químicos de los tres sitios de muestreo. Los valores fueron normalizados y estandarizados y se trabajó con el coeficiente de correlación.

Con el fin de examinar la relación entre componente 1 del ACP-3 y las variables bióticas (riqueza específica, densidad, diversidad, equitatividad y bio- masa expresada como clorofila $a$ ) se efectuó un análisis de regresión $(p<0.05)$, mediante el programa estadístico SPSS (versión 10.0) para Windows.

\section{RESULTADOS}

\section{Variables ambientales}

La Tabla 1 muestra los valores mínimos (m), máximos $(\mathrm{M})$, promedios $(\bar{x})$ y desviación standard (DS) de las distintas variables estudiadas.

En la zona limnética, se observó un máximo registro de profundidad hacia comienzos del otoño y una diferencia de aproximadamente cuatro metros en relación al mínimo valor obtenido (Fig. 2). En las desembocaduras de los ríos la profundidad no superó los $4.6 \mathrm{~m}$. La transparencia en el lago fluctuó entre 0.76-1.43 m (Fig. 2) y en los tributarios el valor máximo fue de $1.45 \mathrm{~m}$ para junio y diciembre/01 en el río El Mollar, observándose los menores registros en el verano en ambas desembocaduras. La relación $\mathrm{Z}_{\mathrm{m}} / \mathrm{Z}_{\mathrm{eu}}$ fluctuó entre 4.1 y 6.8 .

La temperatura del agua, presentó en general un perfil vertical bastante homogéneo, con escasa variación térmica. Durante diciembre/00-febrero y en octubre- diciembre/01 se registraron zonas de discontinuidad a una profundidad no superior al metro y con diferencias máximas de $4{ }^{\circ} \mathrm{C}$ entre la superficie y el fondo (Fig. 3A). Esto

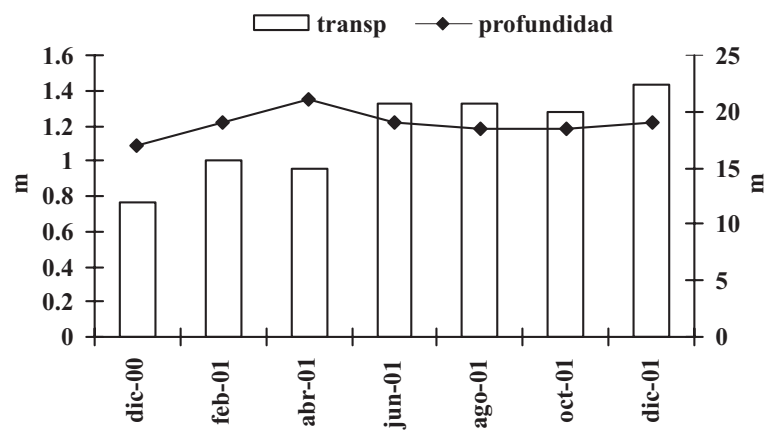

Figura 2. Variaciones de la transparencia (disco de Secchi) y profundidad en la zona limnética del embalse La Angostura. Transparency variations (Secchi disc) and depth in the limnetic zone of the La Angostura dam. 
Tabla 1. Valores mínimos $(\mathrm{m})$, máximos $(\mathrm{M})$ y promedios $(\overline{\mathrm{x}})$ y desviación estándar $(\mathrm{SD})$ de los parámetros físico-químicos y biológicos en la zona limnética y de las desembocaduras de los ríos Tafí y Mollar. Minimum (m), Maximum $(M)$ and average $(\bar{x})$ values and standard deviation (SD) of the physicochemical and biological parameters in the limnetic zone and the estuaries of the Tafi and El Mollar Rivers.

\begin{tabular}{|c|c|c|c|c|c|c|c|c|c|c|c|c|}
\hline & \multicolumn{4}{|c|}{ Zona limnética } & \multicolumn{4}{|c|}{ Desembocadura Río Tafí } & \multicolumn{4}{|c|}{ Desembocadura Río El Mollar } \\
\hline & $\mathrm{m}$ & M & $\bar{x}$ & DS & $\mathrm{m}$ & M & $\bar{x}$ & DS & $\mathrm{m}$ & M & $\bar{x}$ & DS \\
\hline Profundidad (m) & 17 & 21 & 19 & 1.6 & 1 & 3.60 & 2.65 & 1.14 & 3 & 4.6 & 4 & 0.5 \\
\hline Temperatura $\left({ }^{\circ} \mathrm{C}\right)$ & 9 & 26 & 17 & 4.6 & 10 & 24 & 17.8 & 5.1 & 10 & 24 & 18.2 & 4.7 \\
\hline Transparencia (m) & 0.76 & 1.43 & 1.15 & 0.23 & 0.66 & 1.33 & 0.98 & 0.29 & 0.63 & 1.45 & 1.19 & 0.3 \\
\hline $\mathrm{pH}$ & 7.2 & 8.45 & 7.9 & 0.33 & 8.10 & 9 & 8.5 & 0.30 & 8.1 & 8.95 & 8.6 & 0.25 \\
\hline Conductividad eléctrica $(\mu \mathrm{S} / \mathrm{cm})$ & 194 & 408 & 231.7 & 38.5 & 194 & 241 & 210.7 & 16.62 & 188 & 220 & 205.7 & 12.4 \\
\hline Oxígeno disuelto (mg/l) & 2.8 & 7.81 & 5.3 & 1.3 & 5.20 & 7.11 & 6.11 & 0.77 & 2.6 & 7.34 & 5.8 & 1.6 \\
\hline $\mathrm{DBO}_{5}(\mathrm{mg} / \mathrm{l})$ & 0.1 & 1.8 & 0.95 & 0.43 & 0.20 & 2.36 & 1.16 & 0.69 & 0.1 & 1.5 & 0.9 & 0.5 \\
\hline As $(\mu \mathrm{g} / \mathrm{l})$ & 0.02 & 0.03 & 0.02 & 0.005 & 0.015 & 0.02 & 0.02 & 0.003 & 0.025 & 0.04 & 0.03 & 0.006 \\
\hline $\mathrm{Cu}(\mathrm{mg} / \mathrm{l})$ & 0.1 & 4.95 & 1.19 & 1.81 & 0.17 & 4 & 1.61 & 1.98 & 0.1 & 4.8 & 1.11 & 1.84 \\
\hline $\mathrm{Fe}(\mathrm{mg} / \mathrm{l})$ & 0.05 & 0.9 & 0.35 & 0.22 & 0.15 & 0.32 & 0.21 & 0.07 & 0.06 & 0.55 & 0.21 & 0.17 \\
\hline $\operatorname{Mn}(\mathrm{mg} / \mathrm{l})$ & 0.03 & 0.25 & 0.11 & 0.08 & 0.03 & 0.06 & 0.05 & 0.01 & 0.01 & 0.1 & 0.05 & 0.04 \\
\hline $\mathrm{NH}_{4}^{+}(\mathrm{mg} / \mathrm{l})$ & $<0.4$ & $<0.5$ & - & - & $<0.4$ & $<0.5$ & - & - & $<0.4$ & $<0.5$ & - & - \\
\hline $\mathrm{NO}_{2}^{-}(\mathrm{mg} / \mathrm{l})$ & $<0.005$ & $<0.005$ & - & - & $<0.005$ & $<0.005$ & - & - & $<0.005$ & $<0.005$ & - & - \\
\hline $\mathrm{NO}_{3}^{-}(\mathrm{mg} / \mathrm{l})$ & $<0.5$ & $<0.5$ & - & - & $<0.5$ & $<0.5$ & - & - & $<0.5$ & $<0.5$ & - & - \\
\hline $\mathrm{PO}_{4}^{=}(\mathrm{mg} / \mathrm{l})$ & $<0.2$ & $<0.2$ & - & - & $<0.2$ & $<0.2$ & - & - & $<0.2$ & $<0.2$ & - & - \\
\hline $\mathrm{HCO}_{3}^{-}(\mathrm{mg} / \mathrm{l})$ & 98 & 115 & 107 & 6 & & & & & & & & \\
\hline $\mathrm{SO}_{4}^{2-}(\mathrm{mg} / \mathrm{l})$ & 11 & 12 & 11.5 & 0.7 & & & & & & & & \\
\hline $\mathrm{Cl}^{-}(\mathrm{mg} / \mathrm{l})$ & 2.8 & 7.7 & 5.2 & 1.5 & & & & & & & & \\
\hline $\mathrm{Ca}^{2+}(\mathrm{mg} / \mathrm{l})$ & 16 & 21.8 & 19 & 2.3 & & & & & & & & \\
\hline $\mathrm{Mg}^{2+}(\mathrm{mg} / \mathrm{l})$ & 5.4 & 8.36 & 6.31 & & & & & & & & & \\
\hline $\mathrm{Na}^{+}(\mathrm{mg} / \mathrm{l})$ & 17.25 & 19.3 & 18 & 0.8 & & & & & & & & \\
\hline $\mathrm{K}^{+}(\mathrm{mg} / \mathrm{l})$ & 3.7 & 5.1 & 4.4 & 0.6 & & & & & & & & \\
\hline Biomasa (Clorofila $a, \mu \mathrm{g} / \mathrm{l}$ ) & 2.6 & 19 & 10.5 & 4.93 & 10 & 31 & 19.3 & 8 & 6 & 17 & 13 & 3.8 \\
\hline Abundancia algal total (ind/ml) & 50 & 3106 & 659 & 762 & 221 & 2970 & 972 & 9686 & 2382 & 598 & 813 & \\
\hline Diversidad específica & 0.23 & 3 & 1.04 & 0.66 & 0.4 & 1.6 & 0.82 & 0.46 & 0.4 & 1.6 & 0.82 & 0.47 \\
\hline Equitatividad & 0.09 & 0.48 & 0.26 & 0.2 & 0.09 & 0.48 & 0.21 & 0.1 & 0.09 & 0.51 & 0.3 & 0.2 \\
\hline
\end{tabular}

dio como resultado un espesor de la capa de mezcla de aproximadamente 18 metros y una termoclina superficial. La circulación completa de las aguas se manifestó entre abril y agosto (Fig. 3B), coincidente con fenómenos propios de esos meses, donde existe la circulación de fuertes vientos provenientes del norte que alcanzan los $80 \mathrm{~km} / \mathrm{h}$ (EEAOC, 2008). Durante abril se midieron los mínimos registros de temperatura $\left(9^{\circ} \mathrm{C}\right)$.
De acuerdo a las lecturas de $\mathrm{pH}$, las aguas fueron alcalinas, con valores mas elevados de $\mathrm{pH}$ en el estrato superficial y tendencia a la homogeneización durante la circulación (Fig. 3). La conductividad mostró una baja mineralización, con un máximo para la zona limnética, a la profundidad de la lectura de Secchi, en octubre. La predominancia de iones, caracterizaron las aguas como: $\mathrm{HCO}_{3}^{-}>\mathrm{SO}_{4}^{=}>\mathrm{Cl}^{-} ; \mathrm{Ca}^{2+}>\mathrm{Na}^{+}>\mathrm{K}^{+}>\mathrm{Mg}^{2+}$. 


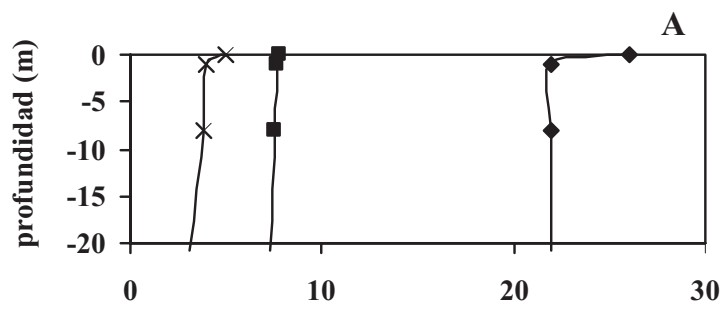

B

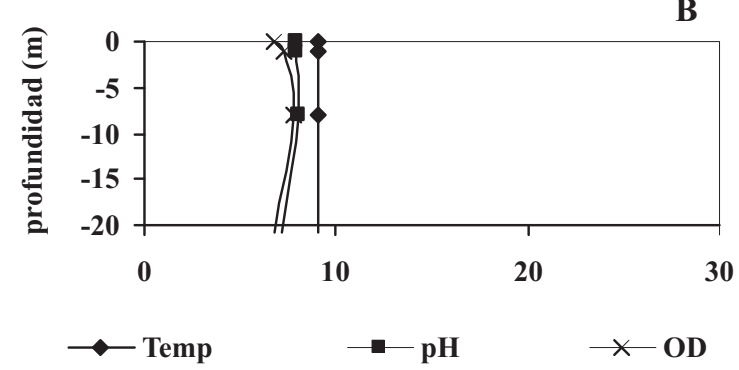

Figura 3. Perfiles de temperatura, (Temp) $\left({ }^{\circ} \mathrm{C}\right), \mathrm{pH}$ y oxígeno disuelto (OD) (mg/l), en la zona limnética. A: período de estratificación (febrero); B: período de mezcla (agosto). Temperature (Temp) $\left({ }^{\circ} \mathrm{C}\right), \mathrm{pH}$ and dissolved oxygen $(\mathrm{DO})(\mathrm{mg} / \mathrm{l})$ profiles in the limnetic zone. A: Stratification period (February); B: mixing period (August).
La metodología utilizada determinó que las concentraciones de nitrógeno como amonio, nitrito y nitrato y el fósforo (como ortofosfato) variaron en un rango no detectable por la metodología utilizada.

El análisis de metales pesados determinó la presencia de arsénico, hierro, cobre y manganeso. Estos dos últimos, en la zona limnética, obtuvieron sus mayores valores en profundidad (Fig. 4 C-D), mientras que para los primeros se detectaron máximos registros en superficie: en abril y junio para el arsénico y en agosto y octubre para el hierro (Fig. 4 A-B). El hierro fue encontrado en los tres sitios, el arsénico estuvo ausente en diciembre/00, el cobre no fue detectado en abril y junio y en muy bajas concentraciones a partir de agosto. El manganeso no fue registrado en superficie de la zona limnética y en las desembocaduras para los meses diciembre/00 y febrero (Fig. 4 A-D).

El oxígeno disuelto disminuyó en la zona limnética hacia el fondo durante los períodos estratificados (Fig. 3A). En las desembocaduras el mínimo valor se obtuvo en diciembre/01 para el
A
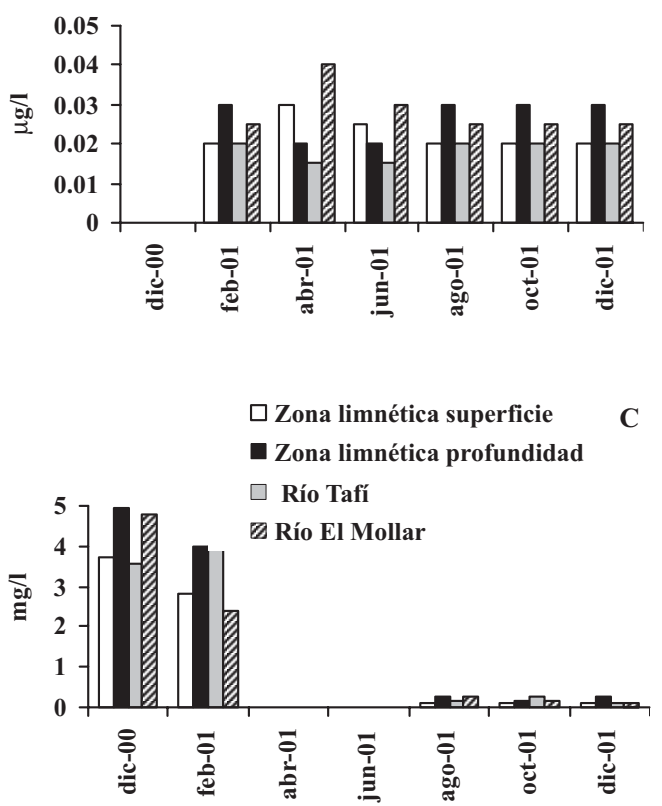

B

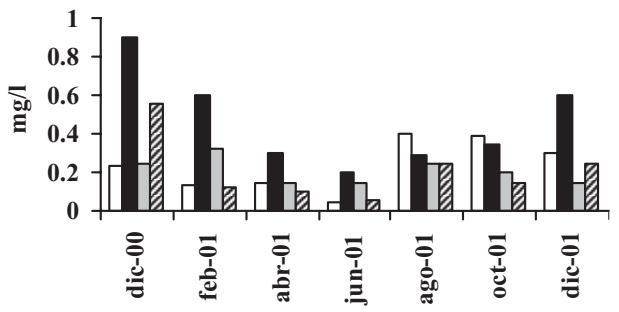

D

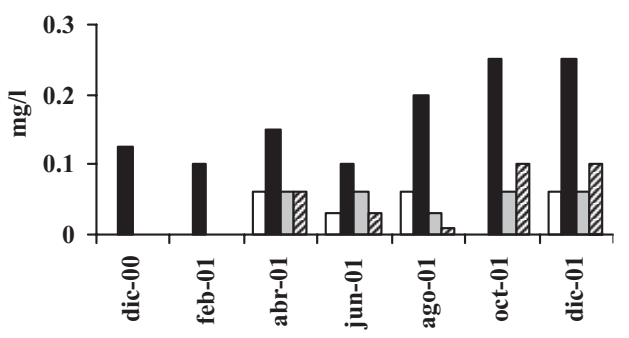

Figura 4. Fluctuaciones de los metales pesados: A: arsénico, B: hierro, C: cobre y D: manganeso en la zona limnética (superficie y fondo) y desembocaduras río Tafí y El Mollar. Fluctuation of heavy metals: arsenic (A), iron (B), copper $(C)$, and manganese $(D)$ in the limnetic zone (surface and bottom) and estuaries of the Tafi and El Mollar Rivers. 
Tabla 2. Abundancia del fitoplancton (ind/ml) en la zona limnética (L) y en las desembocaduras de los ríos El Mollar (M) y Tafí (T). Phytoplankton abundance (ind/ml) in the limnetic zone $(L)$ and the estuaries of the El Mollar $(M)$ and Tafi $(T)$ Rivers.

\begin{tabular}{|c|c|c|c|c|c|c|c|c|c|c|c|c|c|c|c|c|c|c|c|c|c|}
\hline \multirow[t]{2}{*}{ Especies } & \multicolumn{3}{|c|}{ dic-00 } & \multicolumn{3}{|c|}{ feb-01 } & \multicolumn{3}{|c|}{ abr-01 } & \multicolumn{3}{|c|}{ jun-01 } & \multicolumn{3}{|c|}{ ago-01 } & \multicolumn{3}{|c|}{ oct-01 } & \multicolumn{3}{|c|}{ dic-01 } \\
\hline & $\mathrm{L}$ & M & $\mathrm{T}$ & $\mathrm{L}$ & M & $\mathrm{T}$ & $\mathrm{L}$ & M & $\mathrm{T}$ & $\mathrm{L}$ & M & $\mathrm{T}$ & $\mathrm{L}$ & M & $\mathrm{T}$ & $\mathrm{L}$ & M & $\mathrm{T}$ & $\mathrm{L}$ & M & $\mathrm{T}$ \\
\hline \multicolumn{22}{|l|}{ CYANOPHYTA } \\
\hline \multicolumn{22}{|l|}{ CYANOPHYCEAE } \\
\hline \multicolumn{22}{|l|}{ CHROOCOCCALES } \\
\hline Chroococcus minutus (Kütz.) Nägelli & 0 & 0 & 0 & 1 & 2 & 0 & 2 & 3 & 0 & 2 & 3 & 0 & 1 & 0 & 0 & 2 & 6 & 5 & 0 & 3 & 2 \\
\hline Gomphosphaeria aponina Kützing & 261 & 260 & 136 & 174 & 592 & 631 & 135 & 197 & 191 & 285 & 264 & 256 & 554 & 141 & 962 & 2042 & 2290 & 2805 & 896 & 3 & 1155 \\
\hline Microcystis aeruginosa Kützing & 0 & 0 & 0 & 0 & 0 & 0 & 0 & 0 & 0 & 1 & 0 & 0 & 0 & 0 & 0 & 0 & 0 & 0 & 0 & 0 & 0 \\
\hline $\begin{array}{l}\text { Microcystis pulverea (Good.) Forti } \\
\text { NOSTOCALES }\end{array}$ & 0 & 0 & 0 & 0 & 0 & 0 & 1 & 0 & 0 & 1 & 0 & 0 & 0 & 0 & 0 & 1 & 0 & 0 & 0 & 0 & 0 \\
\hline Anabaena sp. & 0 & 0 & 0 & 0 & 0 & 0 & 0 & 0 & 0 & 0 & 0 & 0 & 0 & 0 & 0 & 1 & 0 & 0 & 1 & 0 & 0 \\
\hline Subtotales & 261 & 260 & 136 & 175 & 594 & 631 & 138 & 200 & 191 & 289 & 267 & 256 & 555 & 141 & 962 & 2045 & 2296 & 2810 & 897 & 6 & 1155 \\
\hline EUGLENOPHYTA & & & & & & & & & & & & & & & & & & & & & \\
\hline EUGLENOPHYCEAE & & & & & & & & & & & & & & & & & & & & & \\
\hline EUGLENALES & & & & & & & & & & & & & & & & & & & & & \\
\hline Euglena sp. & 0 & 0 & 0 & 0 & 1 & 2 & 0 & 0 & 0 & 0 & 0 & 4 & 0 & 0 & 0 & 8 & 0 & 88 & 0 & 0 & 26 \\
\hline Trachelomonas sculpta Balech & 2 & 0 & 0 & 2 & 1 & 0 & 4 & 0 & 0 & 1 & 2 & 0 & 3 & 0 & 0 & 5 & 16 & 0 & 6 & 0 & 0 \\
\hline Trachelomonas westii Wolosz & 0 & 0 & 11 & 1 & 0 & 4 & 0 & 0 & 4 & 3 & 0 & 1 & 0 & 0 & 4 & 3 & 12 & 19 & 6 & 0 & 8 \\
\hline Subtotales & 2 & $\mathbf{0}$ & 11 & 3 & 2 & 6 & 4 & $\mathbf{0}$ & 4 & 4 & 2 & 5 & 3 & $\mathbf{0}$ & 4 & 16 & 28 & 107 & 12 & $\mathbf{0}$ & 34 \\
\hline DINOPHYTA & & & & & & & & & & & & & & & & & & & & & \\
\hline DINOPHYCEAE & & & & & & & & & & & & & & & & & & & & & \\
\hline PERIDINIALES & & & & & & & & & & & & & & & & & & & & & \\
\hline Ceratium hirundinella (O. F. Müller) & 0 & 0 & 0 & 0 & 0 & 0 & 1 & 4 & 10 & 14 & 25 & 29 & 5 & 0 & 8 & 0 & 5 & 5 & 13 & 0 & 61 \\
\hline Dujardin tipo robustum & & & & & & & & & & & & & & & & & & & & & \\
\hline Peridinium willei $\mathrm{f}$. lineatum & 0 & 0 & 0 & 0 & 0 & 0 & 0 & 0 & 0 & 0 & 1 & 0 & 0 & 0 & 0 & 11 & 1 & 6 & 0 & 0 & 10 \\
\hline Lindemann & & & & & & & & & & & & & & & & & & & & & \\
\hline Subtotales & $\mathbf{0}$ & $\mathbf{0}$ & $\mathbf{0}$ & $\mathbf{0}$ & $\mathbf{0}$ & $\mathbf{0}$ & 1 & 4 & 10 & 14 & 26 & 29 & 5 & $\mathbf{0}$ & 8 & 11 & 6 & 11 & 13 & $\mathbf{0}$ & 71 \\
\hline CHLOROPHYTA & & & & & & & & & & & & & & & & & & & & & \\
\hline CHLOROPHYCEAE & & & & & & & & & & & & & & & & & & & & & \\
\hline CHLOROCOCCALES & & & & & & & & & & & & & & & & & & & & & \\
\hline Ankistrodesmus fusiformis Corda & 0 & 0 & 1 & 1 & 1 & 0 & 0 & 0 & 0 & 0 & 0 & 0 & 0 & 0 & 0 & 1 & 1 & 0 & 0 & 0 & 1 \\
\hline Ankistrodesmus spiralis (Turn.) & 0 & 0 & 0 & 0 & 0 & 1 & 0 & 0 & 0 & 1 & 0 & 0 & 0 & 0 & 0 & 3 & 9 & 5 & 2 & 0 & 1 \\
\hline Lemmermann & & & & & & & & & & & & & & & & & & & & & \\
\hline Botryococcus braunii Kützing & 4 & 1 & 0 & 0 & 0 & 0 & 0 & 0 & 1 & 0 & 0 & 0 & 1 & 1 & 3 & 1 & 1 & 0 & 0 & 0 & 0 \\
\hline Chlamydomonas globosa Snow & 13 & 0 & 0 & 0 & 0 & 0 & 0 & 0 & 0 & 0 & 0 & 0 & 0 & 0 & 0 & 0 & 0 & 0 & 0 & 0 & 0 \\
\hline Coelastrum pseudomicroporum & 0 & 1 & 0 & 0 & 0 & 0 & 1 & 4 & 1 & 2 & 1 & 0 & 1 & 1 & 1 & 0 & 0 & 0 & 0 & 0 & 0 \\
\hline Korshikov & & & & & & & & & & & & & & & & & & & & & \\
\hline Crucigenia quadrata Morren & 65 & 101 & 85 & 2 & 5 & 4 & 3 & 4 & 4 & 2 & 1 & 1 & 1 & 0 & 0 & 2 & 2 & 2 & 2 & 0 & 3 \\
\hline Dictyosphaerium elongatum Hindák & 0 & 0 & 0 & 1 & 0 & 0 & 2 & 4 & 0 & 5 & 0 & 0 & 4 & 5 & 0 & 6 & 3 & 0 & 1 & 0 & 0 \\
\hline Dictyosphaerium pulchellum Wood & 8 & 3 & 6 & 3 & 1 & 0 & 4 & 0 & 2 & 8 & 0 & 1 & 8 & 6 & 6 & 3 & 5 & 4 & 1 & 0 & 2 \\
\hline Kirchneriella lunaris (Kirchn.) & 1 & 0 & 0 & 3 & 0 & 1 & 0 & 0 & 0 & 0 & 0 & 0 & 0 & 0 & 0 & 0 & 0 & 0 & 0 & 0 & 0 \\
\hline Moebius & & & & & & & & & & & & & & & & & & & & & \\
\hline Monoraphidium arcuatum (Kors.) & 1 & 0 & 0 & 21 & 46 & 6 & 1 & 1 & 0 & 11 & 0 & 0 & 0 & 0 & 3 & 2 & 1 & 0 & 0 & 5 & \\
\hline Hindák & & & & & & & & & & & & & & & & & & & & & \\
\hline Monoraphidium sp. & 4 & 0 & 0 & 1 & 0 & 0 & 0 & 0 & 0 & 0 & 0 & 0 & 0 & 0 & 0 & 0 & 0 & 0 & 0 & 0 & 0 \\
\hline Oocystis borgei Snow & 35 & 11 & 4 & 2 & 0 & 0 & 2 & 3 & 0 & 5 & 3 & 0 & 5 & 7 & 7 & 1 & 0 & 0 & 1 & 0 & 1 \\
\hline Oocystis solitaria Wittrock & 0 & 0 & 0 & 0 & 0 & 3 & 0 & 2 & 0 & 1 & 0 & 0 & 0 & 2 & 0 & 0 & 1 & 0 & 1 & 0 & 1 \\
\hline Oocystis taenoensis Komárek & 9 & 0 & 0 & 2 & 3 & 2 & 2 & 1 & 0 & 1 & 3 & 2 & 3 & 0 & 0 & 2 & 1 & 1 & 1 & 0 & 2 \\
\hline Pediastrum duplex var. gracillimum & 0 & 0 & 0 & 0 & 0 & 0 & 1 & 0 & 0 & 0 & 0 & 0 & 0 & 0 & 0 & 0 & 1 & 0 & 0 & 0 & 0 \\
\hline W. \& G. S. West & & & & & & & & & & & & & & & & & & & & & \\
\hline Pediastrum tetras (Ehrnb.) Ralfs & 0 & 0 & 0 & 0 & 2 & 0 & 0 & 0 & 0 & 1 & 0 & 0 & 0 & 0 & 0 & 0 & 0 & 0 & 0 & 0 & 1 \\
\hline Scenedesmus alternans Hortobagyi & 0 & 0 & 0 & 1 & 1 & 0 & 0 & 0 & 0 & 0 & 0 & 0 & 0 & 0 & 0 & 2 & 0 & 0 & 0 & 0 & 0 \\
\hline Scenedesmus intermedius Chodat & 1 & 0 & 0 & 0 & 1 & 0 & 0 & 0 & 0 & 5 & 0 & 0 & 0 & 0 & 0 & 1 & 0 & 0 & 0 & 0 & 0 \\
\hline Scenedesmus quadricauda (Turpin) & 0 & 0 & 0 & 4 & 0 & 0 & 4 & 2 & 0 & 1 & 3 & 0 & 2 & 0 & 0 & 1 & 0 & 0 & 0 & 0 & 0 \\
\hline Brébisson & & & & & & & & & & & & & & & & & & & & & \\
\hline Sphaerocystis schroeteri Chodat & 5 & 5 & 3 & 2 & 0 & 2 & 6 & 5 & 0 & 1 & 0 & 0 & 2 & 0 & 2 & 0 & 0 & 0 & 1 & 0 & 0 \\
\hline
\end{tabular}


Tabla 2. (continuación)

\begin{tabular}{|c|c|c|c|c|c|c|c|c|c|c|c|c|c|c|c|c|c|c|c|c|c|}
\hline \multirow[t]{2}{*}{ Especies } & \multicolumn{3}{|c|}{ dic-00 } & \multicolumn{3}{|c|}{ feb-01 } & \multicolumn{3}{|c|}{ abr-01 } & \multicolumn{3}{|c|}{ jun-01 } & \multicolumn{3}{|c|}{ ago-01 } & \multicolumn{3}{|c|}{ oct-01 } & \multicolumn{3}{|c|}{ dic-01 } \\
\hline & $\mathrm{L}$ & M & $\mathrm{T}$ & $\mathrm{L}$ & M & $\mathrm{T}$ & $\mathrm{L}$ & M & $\mathrm{T}$ & $\mathrm{L}$ & M & $\mathrm{T}$ & $\mathrm{L}$ & M & $\mathrm{T}$ & $\mathrm{L}$ & $\mathrm{M}$ & $\mathrm{T}$ & $\mathrm{L}$ & M & $\mathrm{T}$ \\
\hline \multicolumn{22}{|l|}{ CHAROPHYCEAE } \\
\hline \multicolumn{22}{|l|}{ ZYGNEMATALES } \\
\hline Closterium aciculare $\mathrm{T}$. West & 1 & 0 & 0 & 1 & 1 & 0 & 7 & 8 & 4 & 2 & 0 & 1 & 10 & 0 & 6 & 1 & 1 & 2 & 2 & 0 & 2 \\
\hline $\begin{array}{l}\text { Closterium acutum var. variabile } \\
\text { (Lemm.) Krieger }\end{array}$ & 7 & 3 & 5 & 1 & 2 & 3 & 2 & 1 & 1 & 0 & 0 & 0 & 4 & 0 & 0 & 2 & 1 & 2 & 4 & 0 & 4 \\
\hline Staurastrum sp. 1 & 0 & 0 & 0 & 0 & 0 & 0 & 1 & 0 & 2 & 2 & 1 & 1 & 7 & 4 & 9 & 2 & 2 & 2 & 2 & 3 & 2 \\
\hline Staurastrum sp. 2 & 0 & 0 & 0 & 0 & 0 & 0 & 1 & 0 & 0 & 1 & 0 & 0 & 5 & 0 & 4 & 2 & 0 & 2 & 1 & 1 & 1 \\
\hline Staurodesmus sp. & 0 & 0 & 0 & 0 & 0 & 0 & 0 & 0 & 0 & 0 & 0 & 0 & 0 & 0 & 0 & 11 & 14 & 0 & 5 & 0 & 0 \\
\hline Subtotales & 154 & 125 & 105 & 49 & 67 & 23 & 39 & 36 & 16 & 39 & 14 & 6 & 71 & 27 & 38 & 47 & 47 & 25 & 25 & 4 & 26 \\
\hline \multicolumn{22}{|l|}{ HETEROKONTOPHYTA } \\
\hline \multicolumn{22}{|l|}{ BACILLARIOPHYCEAE } \\
\hline Aulacoseira granulata(Ehr.) & 0 & 0 & 0 & 4 & 5 & 1 & 4 & 0 & 0 & 0 & 0 & 1 & 1 & 0 & 0 & 10 & 11 & 5 & 7 & 0 & 7 \\
\hline \multicolumn{22}{|l|}{ Simonsen } \\
\hline Cyclotella $\mathrm{sp}$. & 0 & 0 & 0 & 2 & 1 & 0 & 9 & 0 & 0 & 1 & 0 & 0 & 0 & 0 & 0 & 1 & 0 & 2 & 0 & 0 & 0 \\
\hline \multicolumn{22}{|l|}{ BACILLARIALES } \\
\hline Cymbella sp. & 0 & 2 & 0 & 0 & 0 & 0 & 0 & 0 & 0 & 0 & 0 & 0 & 0 & 0 & 0 & 0 & 0 & 0 & 0 & 0 & 0 \\
\hline Diatoma vulgare Bory & 0 & 2 & 0 & 1 & 0 & 0 & 1 & 0 & 0 & 1 & 0 & 0 & 0 & 0 & 0 & 58 & 0 & 0 & 6 & 0 & 0 \\
\hline Ulnaria ulna (Nitzsch) Compère & 0 & 3 & 0 & 0 & 1 & 0 & 0 & 0 & 0 & 0 & 0 & 0 & 0 & 0 & 0 & 0 & 0 & 0 & 0 & 0 & 0 \\
\hline Subtotales & $\mathbf{0}$ & 7 & $\mathbf{0}$ & 7 & 7 & 1 & 14 & $\mathbf{0}$ & $\mathbf{0}$ & 2 & $\mathbf{0}$ & 1 & 1 & $\mathbf{0}$ & $\mathbf{0}$ & 69 & 11 & 7 & 13 & $\mathbf{0}$ & 7 \\
\hline \multicolumn{22}{|l|}{ SYNUROPHYCEAE } \\
\hline \multirow[t]{2}{*}{ Mallomonas sp. } & 0 & 0 & 0 & 1 & 0 & 0 & 0 & 0 & 0 & 0 & 0 & 0 & 0 & 0 & 0 & 0 & 0 & 0 & 0 & 0 & 0 \\
\hline & $\mathbf{0}$ & $\mathbf{0}$ & $\mathbf{0}$ & 1 & $\mathbf{0}$ & $\mathbf{0}$ & $\mathbf{0}$ & $\mathbf{0}$ & $\mathbf{0}$ & $\mathbf{0}$ & $\mathbf{0}$ & $\mathbf{0}$ & $\mathbf{0}$ & $\mathbf{0}$ & $\mathbf{0}$ & $\mathbf{0}$ & $\mathbf{0}$ & $\mathbf{0}$ & $\mathbf{0}$ & $\mathbf{0}$ & $\mathbf{0}$ \\
\hline Totales & 417 & 392 & 252 & 235 & 670 & 661 & 196 & 240 & 221 & 348 & 309 & 297 & 635 & 168 & 1012 & 2188 & 2388 & 2960 & 960 & 10 & 1293 \\
\hline
\end{tabular}

río Mollar. La demanda bioquímica de oxígeno $\left(\mathrm{DBO}_{5}\right)$ no superó los $2.36 \mathrm{mg} / \mathrm{l}$ (Tabla 1$)$.

\section{Composición taxonómica}

Se registraron un total de 66 taxones pertenecientes a las clases: Chlorophyceae (24), Charophyceae (8), Cyanophyceae (11), Euglenophyceae (4), Dinophyceae (3), Bacillariophyceae (17), Xanthophyceae (1) y Synurophyceae (1). Le correspondieron 55 especies a la zona limnética (en toda la columna de agua) y 44 a los ríos y en promedio hubo 18 especies por muestra. En los tres sitios muestreados se destacaron las Chlorophyta por su riqueza. Las especies de Synurophyceae y Xanthophyceae fueron exclusivas de la zona limnética, mientras que en ésta estuvieron ausentes Merismopedia sp., Denticula sp., Eunotia sp, Pinnularia sp, Rhopalodia sp. y Surirella ovalis las que sólo se encontraron en los ríos. De las especies identificadas Gomphosphaeria aponina y Sphaerocystis shroeteri estuvieron siempre pre- sentes, mientras que Chroococcus minutus, Ceratium hirundinella tipo robustum, Peridinium willei f. lineatum, Botryococcus braunii, Oocystis borgei, Closterium aciculare, Closterium venus, Staurastrum sp. 1 y 2 y Aulacoseira granulata se encontraron con una frecuencia entre $80-95 \%$. Las especies frecuentes (detectadas en más del $50 \%$ de las muestras), representaron el $26 \%$ del total de taxa determinados. Las fluctuaciones de la riqueza en las tres zonas fue similar, con excepción de diciembre/01, ya que el aumento registrado en el embalse no se correspondió con los descensos ocurridos en las desembocaduras.

\section{Abundancia del fitoplancton}

La comunidad fitoplanctónica del embalse La Angostura estuvo compuesta por un bajo número de especies abundantes, ya que una o dos contribuyeron a más del $80 \%$ de la densidad total y se observó un número elevado de especies raras (más del 50\%). En la Tabla 2 se muestra el 
listado de las especies, si bien sólo se incluyen aquellas que fueron cuantificadas. El promedio de las abundancias de la comunidad fitoplanctónica en la columna de agua presentó un rango de variación entre $179 \mathrm{ind} / \mathrm{ml}$ (abril/01) a $2122 \mathrm{ind} / \mathrm{ml}$ (octubre/01). En la zona limnética el máximo de densidad algal obtenido de la suma de los cuatro niveles ( $8490 \mathrm{ind} / \mathrm{ml}$ ) ocurrió en el período de aguas bajas y medianamente transparentes, durante la primavera (octubre/01). El mismo fue alrededor de 3.6 veces mayor que el promedio anual (2359 ind/ml). En la figura 5A$\mathrm{C}$, se observa la dominancia de algas azules durante todo el año, para los tres sitios muestreados con Gomphosphaeria aponina como responsable. Los mínimos de densidad algal ocurrieron durante el período de lluvias (verano y comienzos del otoño). En las desembocaduras, además del máximo observado en la zona limnética para octubre, se dio un segundo incremento en verano
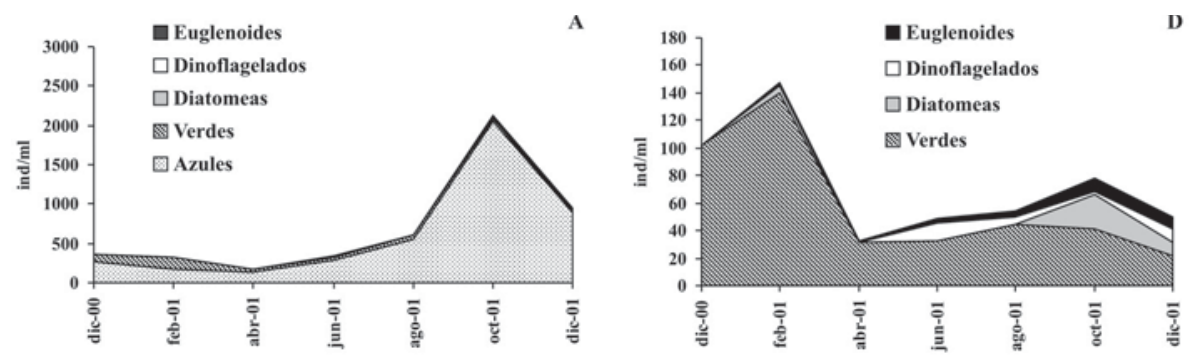

B
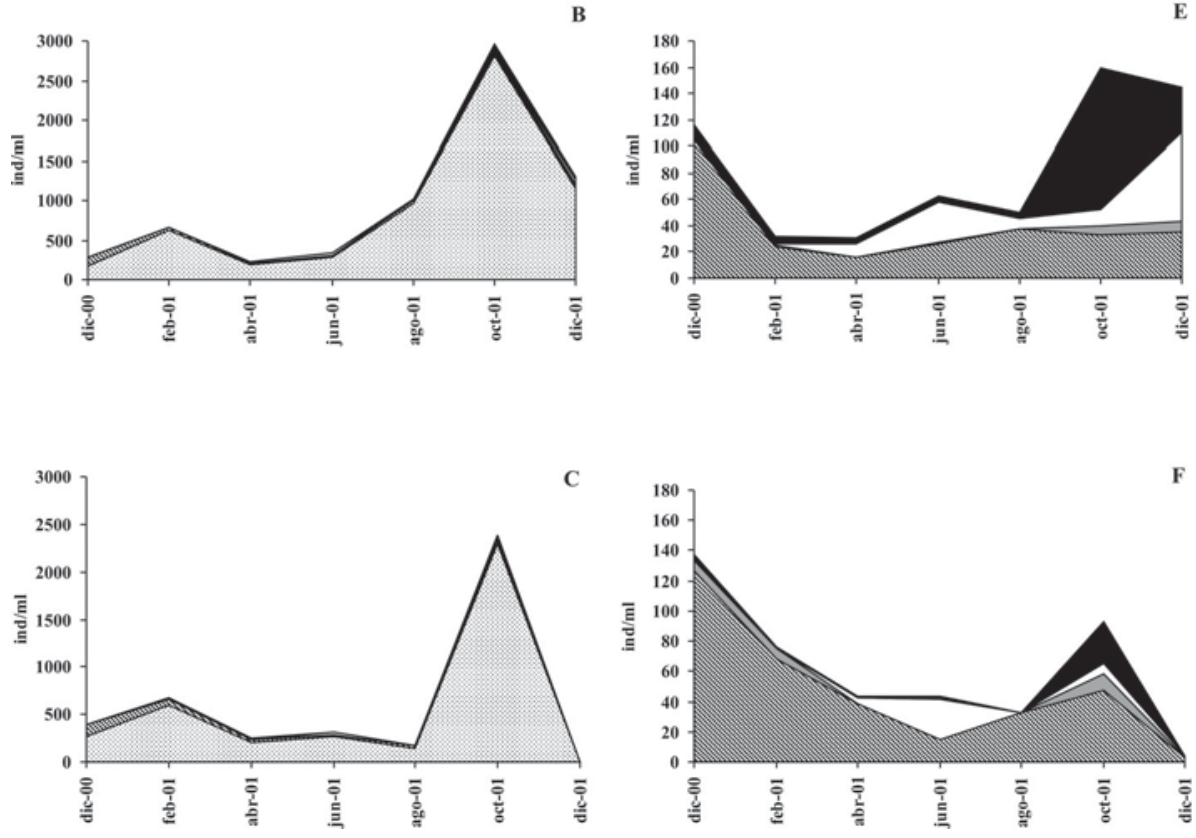

Figura 5. Fluctuaciones de la densidad fitoplanctónica. A-C: incluyendo cianobacterias: A: Zona limnética (valores promedios de la columna de agua); B: Desembocadura río Tafí; C: Desembocadura río El Mollar. D-F: excluyendo las cianobacterias: D: Zona limnética (valores promedios de la columna de agua); E: Desembocadura río Tafí; F: Desembocadura río El Mollar. Fluctuations in phytoplankton density. A-C: including cyanobacteria. A: limnetic zone (average values of the water column), B: Tafi River estuary, C: El Mollar River estuary. D-F: excluding cyanobacteria: D: limnetic zone (water column average values), E: Tafi River estuary, F: El Mollar River estuary. 


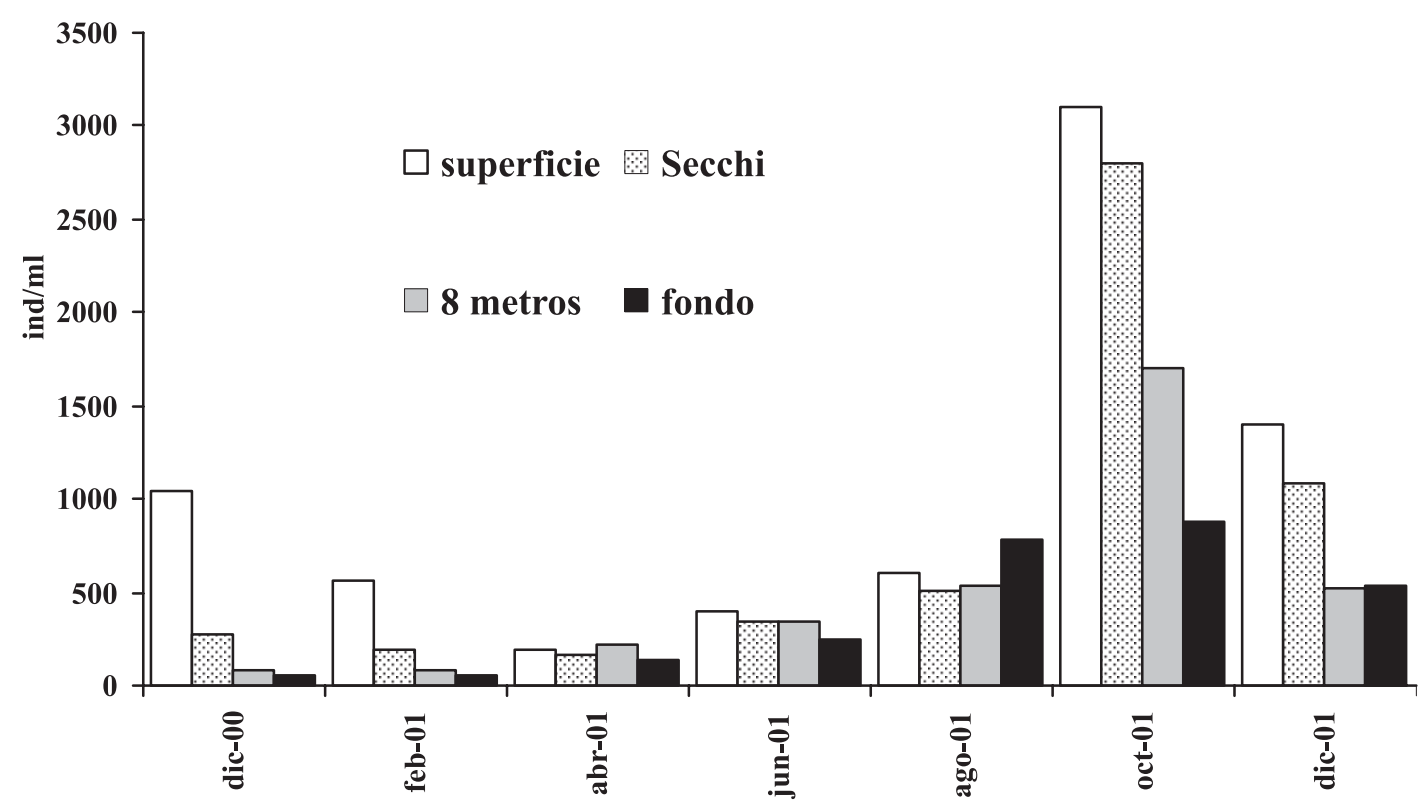

Figura 6. Densidad fitoplanctónica en la zona limnética para los distintos niveles de la columna de agua. Phytoplankton density in the limnetic zone for the different levels of the water column.

(período de aguas altas), también con predominio de algas azules. La figura 5D-F, muestra la fluctuación de las densidades de los otros grupos algales exceptuando las cianobacterias: en general las abundancias de las azules fueron seguidas por las verdes. En la zona limnética las diatomeas se destacaron en octubre y los dinoflagelados en junio. En las desembocaduras de los ríos los euglenoides se distinguieron en octubre y las dinofitas en junio para ambas y también en diciembre/01 para el Tafí. En la zona limnética las especies Crucigenia quadrata, Monoraphidium arcuatum, Diatoma vulgare y Ceratium hirundinella tipo robustum se presentaron como subdominates, cuyos valores máximos respectivamente fueron: 95, 58, 54, y 28 ind $/ \mathrm{ml}$. En el Tafí después de G. aponina estuvieron Euglena acus (88 ind/ml), C. quadrata (85 ind $/ \mathrm{ml})$, y C. hirundinella tipo robustum $(61 \mathrm{ind} / \mathrm{ml})$ y en El Mollar C. quadrata $(101 \mathrm{ind} / \mathrm{ml})$ y $M$. arcuatum $(45 \mathrm{ind} / \mathrm{ml})$. En la figura 6, se observa que los máximos registros de densidad algal ocurrieron en general en la zona eufótica (superficie y Secchi), con una disminución hacia el fondo, a excepción de agosto que fue a la inversa.

\section{Biomasa del fitoplancton}

Los valores de biomasa del fitoplancton medida como concentración de clorofila $a$, se observan en la Tabla 1. Los mayores registros se produjeron en abril, a los 8 metros, con $19 \mu \mathrm{g} / \mathrm{l}$ y para febrero en la desembocadura del río Tafí $(31 \mu \mathrm{g} / \mathrm{l})$. En la zona limnética, en períodos estratificados, los mínimos de clorofila $a$ se obtuvieron en el fondo, durante diciembre/00, febrero, octubre y diciembre/01, $2.8 \mu \mathrm{g} / \mathrm{l}, 4 \mu \mathrm{g} / \mathrm{l}$, $2.6 \mu \mathrm{g} / \mathrm{l}$ y $5.6 \mu \mathrm{g} / \mathrm{l}$ respectivamente, mientras que en período de mezcla, en agosto, el máximo se ubicó a los 20 metros $(16.3 \mu \mathrm{g} / \mathrm{l})$.

\section{Diversidad y equitatividad del fitoplancton}

En la figura 7A se observan los valores promedios de diversidad en la columna de agua y de las desembocaduras. Los máximos valores de diversidad en la zona limnética se obtuvieron desde diciembre/00 a abril (febrero con 2, a los $8 \mathrm{~m}$ y en abril en el fondo con 3), con una disminución marcada a partir de agosto, alcanzando un mínimo (Tabla 1) en octubre, a $1.3 \mathrm{~m}$ (profundidad del 
Tabla 3. Coliformes totales (CT), coliformes fecales (CF) y Streptofecales (SF) (UFC/100 ml) en los diferentes niveles de la zona limnética del embalse La Angostura y en la superficie de las desembocaduras de los ríos. Total coliform (TC), faecal coliform (FC), and Streptofecales (FS) in the different levels of the limnetic zone of the La Angostura dam and at the surface of the rivers' estuaries.

\begin{tabular}{|c|c|c|c|c|c|c|c|c|c|c|c|c|c|c|c|c|c|c|c|c|c|}
\hline & \multicolumn{3}{|c|}{ Dic-00 } & \multicolumn{3}{|c|}{ Feb-01 } & \multicolumn{3}{|c|}{ Abr-01 } & \multicolumn{3}{|c|}{ Jun-01 } & \multicolumn{3}{|c|}{ Ago-01 } & \multicolumn{3}{|c|}{ Oct-01 } & \multicolumn{3}{|c|}{ Dic-01 } \\
\hline & $\mathrm{CT}$ & $\mathrm{CF}$ & SF & CT & $\mathrm{CF}$ & SF & CT & $\mathrm{CF}$ & SF & $\mathrm{CT}$ & $\mathrm{CF}$ & SF & $\mathrm{CT}$ & $\mathrm{CF}$ & SF & CT & $\mathrm{CF}$ & SF & $\mathrm{CT}$ & $\mathrm{CF}$ & SF \\
\hline Zona limnética & & & & & & & & & & & & & & & & & & & & & \\
\hline Superficie & 120 & 0 & 0 & 80 & 7 & 10 & 380 & 7 & 120 & 240 & 2 & 120 & 120 & 1 & 40 & 10 & 4 & 5 & 70 & 3 & 0 \\
\hline Profundidad disco de Secchi & 80 & 1 & 0 & 80 & 5 & 20 & 120 & 4 & 50 & 25 & 6 & 2 & 40 & 2 & 3 & 20 & 5 & 8 & 350 & 200 & 20 \\
\hline $8 \mathrm{~m}$ & 170 & 1 & 0 & 250 & 90 & 180 & 60 & 2 & 8 & 320 & 1 & 150 & 20 & 2 & 5 & 15 & 3 & 0 & 75 & 15 & 8 \\
\hline Fondo & 250 & 0 & 0 & 170 & 22 & 350 & 90 & 4 & 30 & 380 & 1 & 80 & 20 & 0 & 50 & 30 & 5 & 70 & 120 & 10 & 15 \\
\hline Desembocadura río Tafí & 60 & 3 & 0 & 430 & 6 & 12 & 140 & 10 & 80 & 870 & 5 & 220 & 40 & 2 & 80 & 30 & 7 & 12 & 50 & 20 & 30 \\
\hline Desembocadura río El Mollar & 45 & 2 & 0 & 160 & 7 & 0 & 29 & 3 & 6 & 6700 & 1 & 1500 & 5 & 1 & 1 & 8 & 3 & 4 & 82 & 15 & 20 \\
\hline
\end{tabular}

disco de Secchi) y a 8 metros. En las desembocaduras, los mayores registros de diversidad se obtuvieron en diciembre/00 y los menores se observaron en febrero, agosto y octubre. Esta variable tuvo una correlación significativa negativa con la abundancia fitplanctónica $\left(r^{2}=0.51, p<0.05\right)$.

Los valores de equitatividad en general fueron bajos (Fig. 7B), los que disminuyeron a partir de ju-
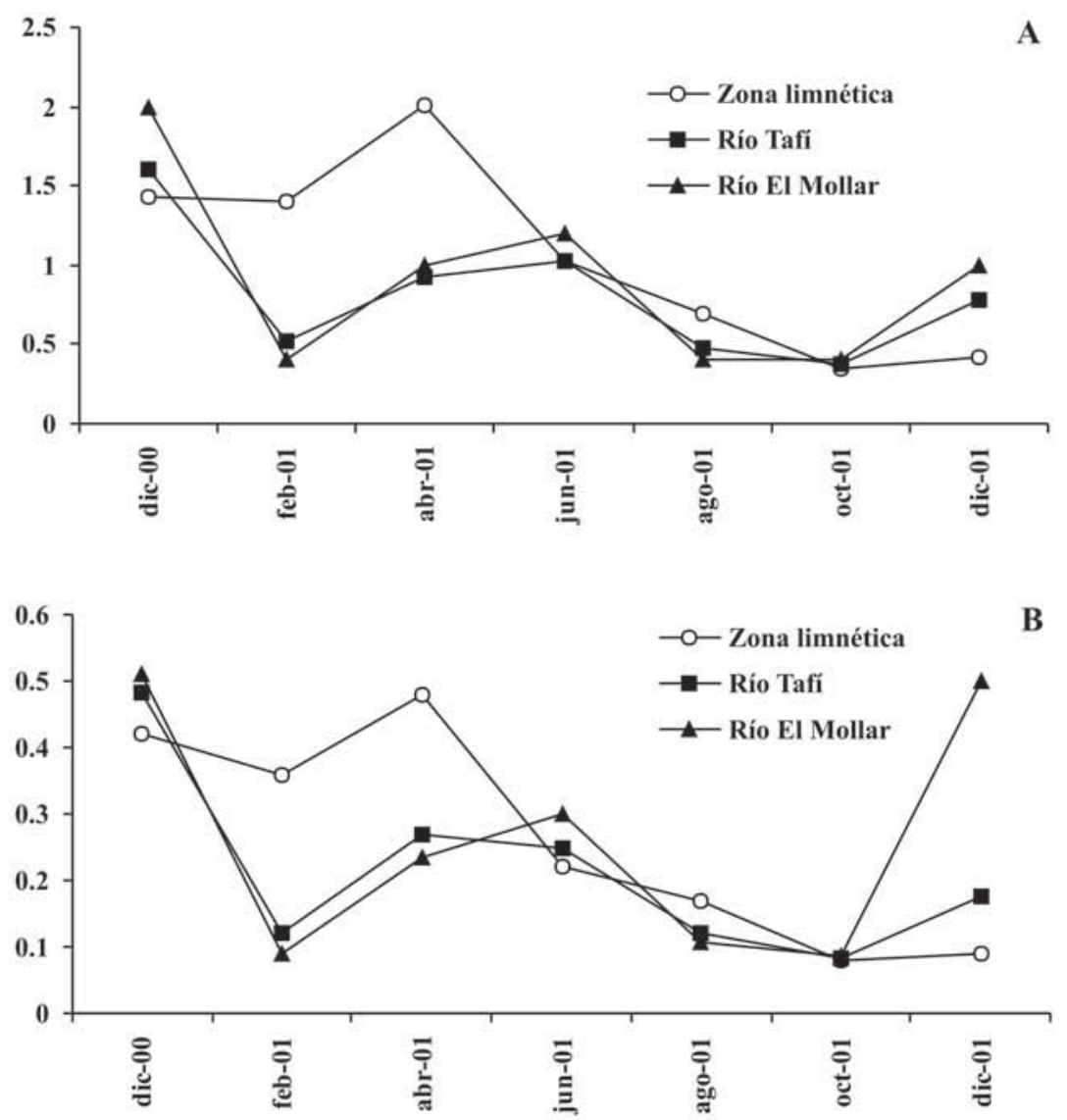

Figura 7. Fluctuaciones de la diversidad específica y equitatividad de los tres sitios. A: Diversidad específica, B: equitatividad. Fluctuations in the specific diversity and equitativity of the three sampling sites. A: specific diversity, B: equitativity. 
nio. Este parámetro mostró una fluctuación similar a la diversidad con excepción de diciembre/01 para el río Mollar, cuando este tributario tuvo muy pocas especies y baja densidad de las mismas.

\section{Análisis Bacteriológicos}

En los análisis bacteriológicos sólo se detectaron en junio valores mayores de $10^{3} \mathrm{UFC} / 100 \mathrm{ml}$ de coliformes totales (CT) y Streptofecales (SF) para la desembocadura del Mollar (Tabla 3).

\section{Análisis de componentes principales}

En la figura 8A se observa la distribución de la muestras en base al análisis de componentes principales (ACP-1) utilizando las densidades algales. El Componente 1 explicó el $27.53 \%$ de la
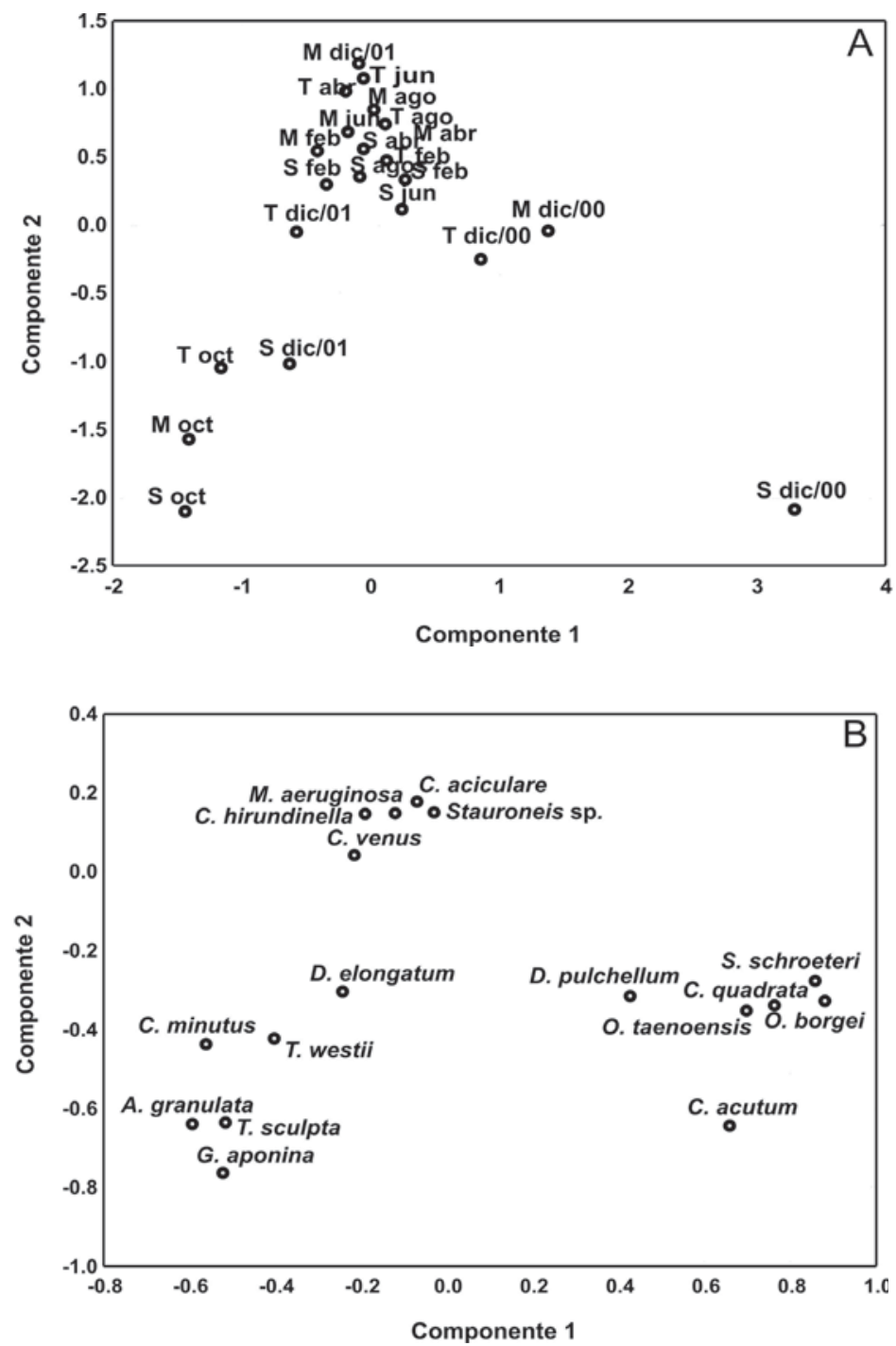

Figura 8. Ordenación de las muestras (A) y de las especies (B) de los tres sitios: zona limnética y desembocaduras de los ríos Tafí y El Mollar, en el espacio formado por los dos primeros ejes del Análisis de Componentes Principales 1 (ACP-1), utilizando las abundancias de las 17 especies del fitoplancton seleccionadas. Ordination of the samples $(A)$ and species $(B)$ of the three sampling sites: limnetic zone and the Tafi and El Mollar River estuaries; in the space created by the two first axes of the Principal Component Analysis 1 (PCA-1), using the abundancy of the 17 selected phytoplankton species. 


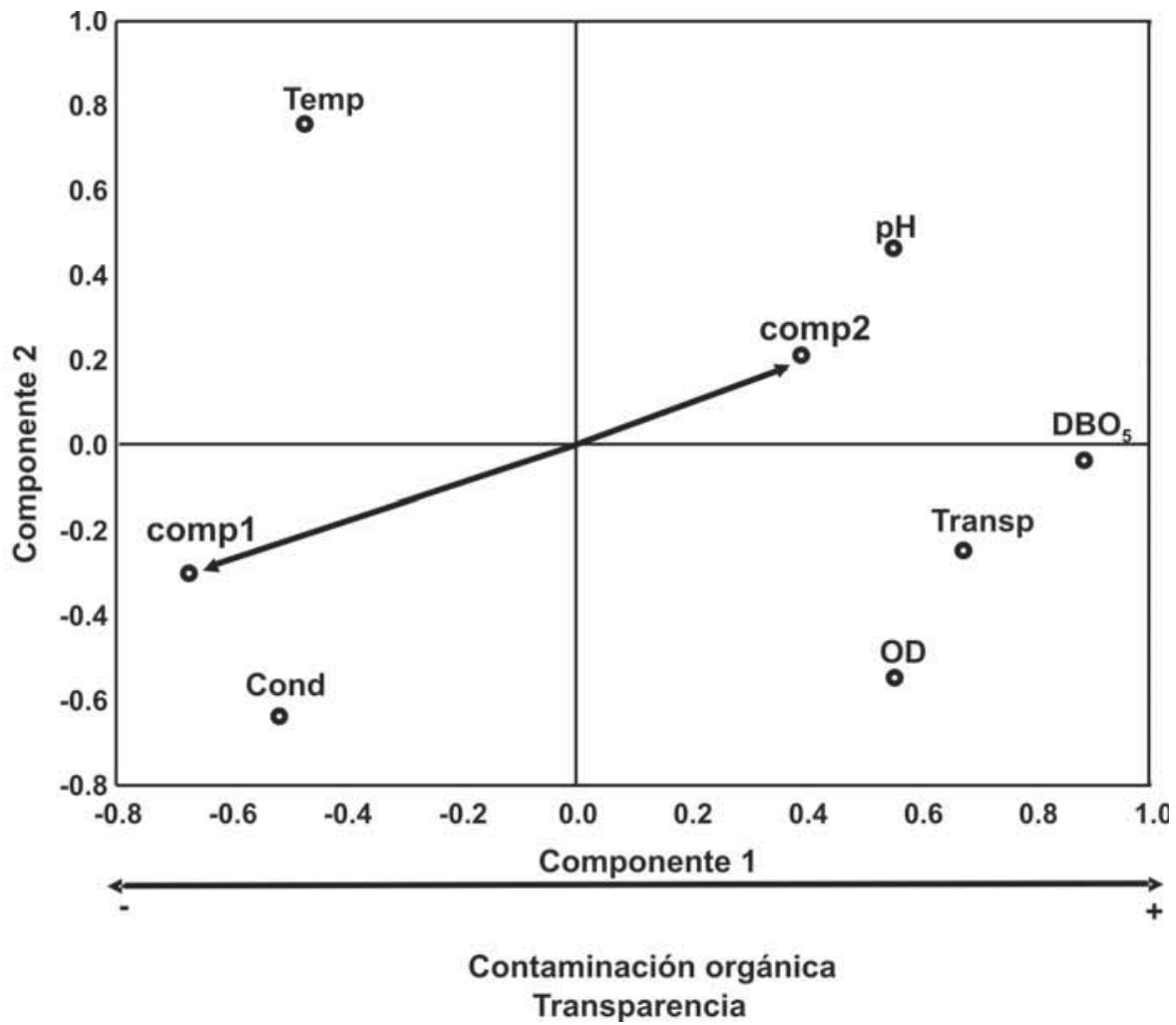

Figura 9. Posición de las variables físico-químicas (temperatura (Temp), transparencia (Transp), pH, conductividad eléctrica (Cond), oxígeno disuelto (OD), $\mathrm{DBO}_{5}$ ) y de los dos primeros componentes del ACP-1 (comp1 y comp2) en el plano definido por los dos primeros componentes del Análisis de Componentes Principales 2 (ACP-2). Position of the physicochemical variables (temperature (Temp), transparency (Transp), $p H$, electrical conductivity (Cond), dissolved oxygen (DO), BOD ), and the first two PCA-1 components (comp1 and comp2) in the plane defined by the first two components of the Principal Component Analysis 2 (PCA-2).

varianza total y el 2 explicó el $17.1 \%$. El ACP mostró que prácticamente no existieron diferencias significativas entre los sitios ni entre los distintos períodos del año, ya que las proporciones de las especies se mantuvieron similares. Sólo las muestras de diciembre/00 y octubre se diferenciaron del resto. Las primeras se caracterizaron por la presencia de Crucigenia quadrata, Sphaerocystis schroeteri, Oocystis borgei, O. taenoensis y Closterium acutum. En octubre las muestras se diferenciaron por las abundancias en particular de Gomphosphaeria aponina, acompañada por otras especies como Aulacoseira granulata, Trachelomonas sculpta, T. westii, Chroococcus minutus y Dictyosphaerium elongatum (Fig. 8B).

Con el objetivo de esclarecer la clasificación preliminar de las muestras se llevó a cabo un segundo análisis de componentes principa- les (ACP-2), incluyendo los dos primeros componentes del ACP-1 junto a las variables ambientales (temperatura, transparencia, conductividad eléctrica, $\mathrm{pH}, \mathrm{OD}$ y $\mathrm{DBO}_{5}$ ) (Fig. 9). Los dos primeros componentes acumularon un $57 \%$ de la variabilidad de las muestras. El primero (comp1), derivado de la abundancia, se relacionó con la conductividad y la temperatura y posee los valores máximos negativos para el componente 1 del ACP-2, mientras que en el extremo del mismo eje se encuentra el componente 2 (comp2), el que se relacionó más con el $\mathrm{pH}$, la transparencia y el OD. De esta manera se pudo observar un gradiente trófico respecto al primer componente y un gradiente térmico en relación a ambos componentes.

El factor 1 derivado del ACP-3 realizado con las variables abióticas incluyendo las muestras 


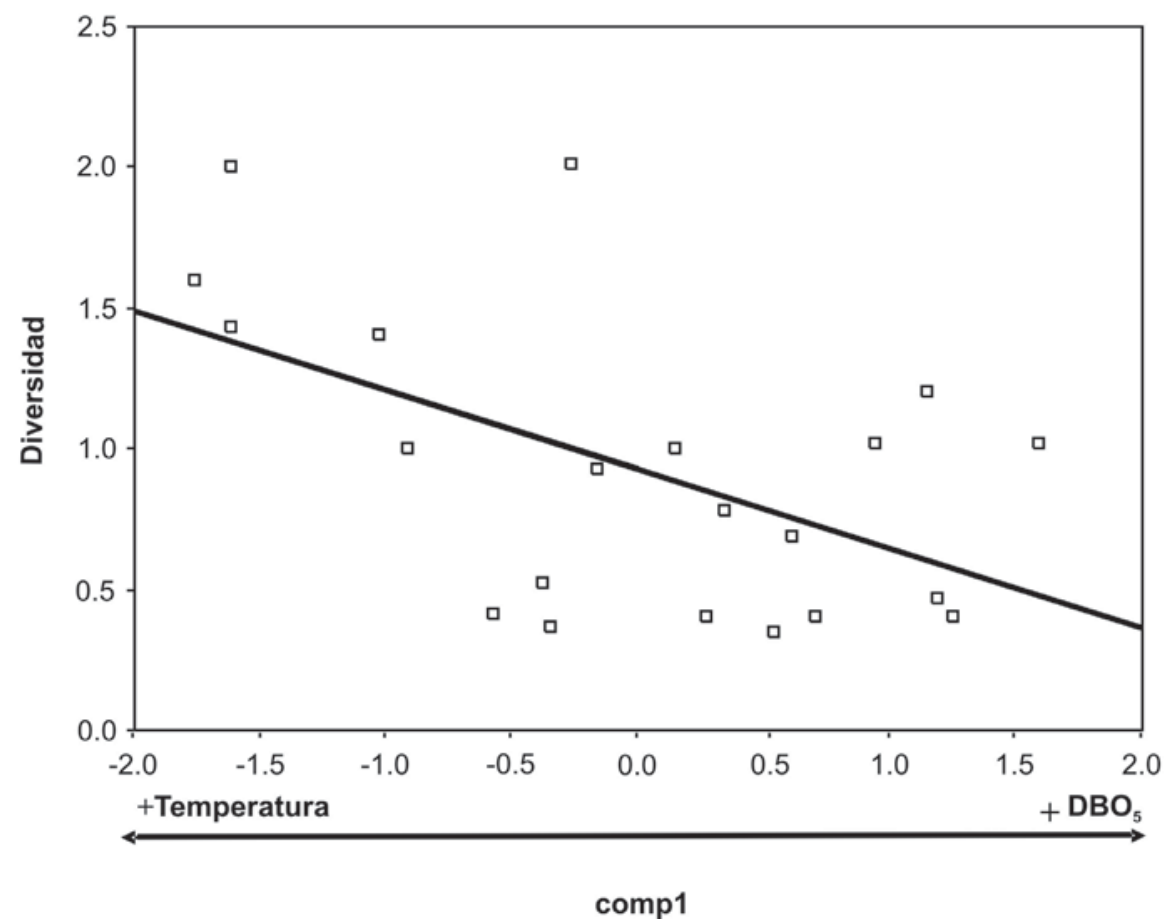

Figura 10. Análisis de regresión entre el componente 1 (comp1) del ACP-3 y la diversidad. Regression analysis between PCA-3 component 1 (compl) and diversity.

de los tres sitios representó un gradiente positivo en relación a la $\mathrm{DBO}_{5}$ y negativo en relación a la temperatura. Del análisis de regresión múltiple entre el factor 1 y las variables abióticas, la $\mathrm{DBO}_{5}$ explicó un $77 \%$ de la variabilidad de este componente y junto a la temperatura un $91 \%$. La regresión lineal entre el factor 1 (del ACP-3) y la diversidad (única variable biótica con resultado significativo) se grafica en la figura $10\left(r^{2}=0.3\right)$. En esta figura se observa que la diversidad tiende a aumentar a mayor temperatura y disminuir con los incrementos de la $\mathrm{DBO}_{5}$.

\section{DISCUSIÓN Y CONCLUSIONES}

Por el comportamiento térmico, el embalse La Angostura se ubica dentro del tipo monomíctico cálido (Margalef, 1983), el que estuvo caracterizado por un período de mezcla durante la estación seca y uno estratificado durante el verano, el que coincidió con las estaciones lluviosas. Las pequeñas diferencias de temperatura en la colum- na de agua conformaron una estratificación débil.

Los resultados de OD y $\mathrm{DBO}_{5}$, comparado con embalses de baja cota de la provincia de Tucumán (Dr. C. Gelsi y Río Hondo), mostraron que La Angostura no presentó contaminación orgánica, ya que estos valores pudieron considerarse aceptables en toda la columna de agua y durante todo el año (Seeligmann \& Tracanna, 1994; Locascio de Mitrovich et al., 1997; Tracanna et al., 1999, 2006, entre otros). Por otra parte, aún cuando La Angostura tiene un espejo de agua considerablemente menor y un tiempo de residencia bajo, en relación con los otros embalses, el número de especies registradas fue similar a las obtenidas en Dr. C. Gelsi y los valores de biomasa (clorofila $a$ ) se asemejaron a los de Río Hondo. Sin embargo, La Angostura se diferenció por el predominio de algas azules.

Por tratarse de un lago subtropical pero emplazado en una zona montañosa, aunque con actividades agrícolas, La Angostura no se ajustó a una categoría trófica definida. Por el contrario, los parámetros tales como disminución de la diversidad a mayor 
abundancia, elevado $\mathrm{pH}$, grandes fluctuaciones de la biomasa fitoplanctónica, y registros menores a 0.005 y $0.5 \mathrm{mg} / \mathrm{l}$ de nitrito y nitrato respectivamente, se lo podría clasificar como eutrófico. Sin embargo, los niveles de clorofila $a, \mathrm{OD}, \mathrm{DBO}_{5} \mathrm{y} \mathrm{NH}_{4}^{+}$ lo ubicarían dentro de un cuerpo de agua del tipo oligo-mesotrófico (Dasí, 1998, Huszar, 1998, Sládecek, 1973, entre otros). La detección puntual de coliformes totales, fecales y Streptofecales en la desembocadura del río El Mollar reflejarían una descarga inusual de residuos no tratados al embalse, por lo que no permitiría ser un indicador adecuado del nivel trófico. Como tampoco lo sería la dominancia casi absoluta de cianobacterias durante el período estudiado, ya que las características térmicas favorecerían las condiciones adecuadas para permitir el crecimientode algas azules durante todo el año (Reynolds, 1998).

En el caso particular de La Angostura, la abundancia de cianobacterias detectadas, se debería a que condiciones de calma y turbulencia permitieron la migración de una población dispersa a la superficie del agua (Reynolds, 1987). El comienzo del crecimiento cianobacterial coincidió con los mínimos valores de la relación $\mathrm{Z}_{\mathrm{m}} / \mathrm{Z}_{\text {eu }}$, en concordancia con los resultados obtenidos por otros autores (Bormans et al., 2005). Según Shapiro (1990), el predominio de las cianobacterias se explicaría en todos los casos por su elevada capacidad para absorber dióxido de carbono disuelto, aun en concentraciones muy bajas. Otro factor que pudo generar el alto desarrollo de G. aponina en el embalse estaría dado por el $\mathrm{pH}$ elevado, ya que éste favorece el crecimiento de las cianobacterias, por su capacidad para transformar los iones bicarbonato y carbonato en dióxido de carbono (Olofsson, 1980; Shapiro, 1990). De hecho, las cianobacterias son las únicas algas que desarrollan biomasas importantes en ambientes naturalmente alcalinos y salinos (Fogg et al., 1976; Skulberg et al., 1984). Además, la baja tasa de filtración por parte del zooplancton, debido que son poco palatables (Shapiro, 1990). Eng-Wilmot et al. (1977), determinaron que G. aponina prolifere durante la primavera tardía y comienzos del verano como también en el otoño cuando las aguas son más cálidas. En general, el cre- cimiento masivo de algas se manifiesta cuando se conjugan una serie de factores como la existencia de una población previa, presencia de organismos con flotabilidad y vesículas de gas y estabilidad en la columna de agua. Este crecimiento de las poblaciones algales puede ocurrir de forma tal que no se haya detectado previamente la presencia manifiesta de los organismos (Reynolds \& Walsby, 1975, entre otros). También es interesante destacar la presencia de $C$. hirundinella t. robustum, ya que aún cuando no alcanzó densidades altas como en otros embalse del país (Rodríguez y Cossavella, 2000; Prosperi, 2000), fue el primer registro de esta especie para la provincia (Seeligmann y Tracanna, 2003). Este dinoflagelado se encontró primeramente en lagos Andino-Patagónicos, notándose posteriormente una distribución hacia el norte argentino, sin una explicación adecuada a dicha propagación. Sin embargo, el desarrollo masivo, casi simultáneo de $C$. hirundinella t. robustum debería estar relacionado a importantes alteraciones ambientales (Boltovskoy et al., 2003).

$\mathrm{Si}$ bien existen aspectos controvertidos respecto a la variación de la diversidad en relación a los diferentes gradientes de contaminación, nuestros resultados concordaron con lo expresado por algunos autores (Margalef, 1969, 1983; Hendey, 1977).

Las concentraciones de $\mathrm{Cu}$ registradas en el embalse se encontrarían en el límite de toxicidad, ya que a partir de los $5 \mathrm{mg} / \mathrm{l}$ se pueden observar disminuciones drásticas de la densidad algal (Cordero et al., 2005). Debido a la detección puntual de este metal se deduce, como lo señalan Isasmendi et al. (2007), que proviene del uso de pesticidas y fertilizantes que contienen entre sus componentes dicho catión. Topalián et al., (1999) proponen una clasificación de los sistemas acuáticos, basada en la concentración de metales pesados. De acuerdo a ella, un cuerpo de agua excesivamente poluído (grado IV) tendría una concentración de $\mathrm{Cu}$ mayor a $20 \mathrm{mg} / \mathrm{l}$.

El ACP-1 permitió inferir que no hubo diferencias destacables entre sitios, pero si temporales. De esta manera pocas especies fueron características o raras y hubo muchas comunes a todas las muestras. La temperatura minimizó el efecto de las otras variables abióticas, por lo que el ACP-2 
no pudo confirmar la interpretación obtenida a partir del ACP-1. El componente 1 del ACP-1 realizó una separación de muestras a través de un gradiente trófico, ubicando en el extremo negativo del eje de las equis a $G$. aponina, A. granulata junto a Euglenophyta durante el comienzo de la estratificación (octubre) y en el positivo especies con menor nivel trófico. El componente 2 del ACP-1 segregó las especies respecto a su forma de vida, ubicando organismos unicelulares y de rápido crecimiento como $C$. venus, $C$. aciculare, $M$. arcuatum en el extremo positivo y agregados celulares hacia el negativo, G. aponina, S. schroeteri, $O$, borgei y $O$. taenoensis, entre otros.

La distribución de las muestras en relación al gradiente de transparencia estuvo principalmente influenciada por el régimen de lluvias. Los mínimos valores de este parámetro se produjeron a fines de la primavera y durante el verano, cuando se registran las máximas precipitaciones, junto a alta turbidez (3.5 UNT) como consecuencia de los sólidos suspendidos arrastrados por los tributarios y altas temperaturas. Los organismos favorecidos por esta compleja situación fueron Chlorophyta de pequeño tamaño y formas coloniales, no habiéndose registrado fitoflagelados. Por el contrario, durante meses más secos, ocurrieron los máximos de transparencia y se destacaron cianobacterias, diatomeas y euglenoides.

Los resultados obtenidos a partir de los análisis multivariantes permitieron confirmar que la composición del fitoplancton estuvo en primer lugar determinada por la temperatura, la que ejerció control sobre mezcla física de la columna de agua. Esta última además, se vio afectada, como factor adicional, por fuertes vientos. En segundo lugar, dependió del ciclo hidrológico ya que fue el parámetro que más influyó sobre la transparencia del agua. Por último, los distintos indicadores tróficos también intervinieron en la dinámica del fitoplancton.

Dado que el embalse fue creado para cubrir necesidades directas con el ser humano (agua para consumo, turismo, industrias, diversificación agropecuaria y recreación), los eventos detectados de cobre y coliformes, acusan la necesidad de realizar estudios de seguimiento a los fines de evitar daños a la biota y la salud humana.

\section{AGRADECIMIENTOS}

Este estudio fue financiado por el Consejo Nacional de Investigaciones Científicas y Técnicas (CONICET), a través del proyecto PIP 0871/98 y el Consejo de Investigaciones de la Universidad Nacional de Tucumán (CIUNT), con los proyectos 26/G229 y G335/1.

\section{BIBLIOGRAFÍA}

APHA. 1992. Standard methods for the examination of water and wastewater. $18^{\text {th }}$ ed. American Public Health Association, Washington. 710 pp.

BOLTOVSKOY, A., R. ECHENIQUE y J. M. GUERRERO. 2003. Colonización de limnótopos de Argentina por Ceratium hirundinella (Dinophyceae). Bol. Soc. Argent. Bot., 38(Supl.): 148.

BORMANS, M., P. W. FORTH \& L. FABBRO. 2005. Spatial and temporal variability in cyanobacterial population controlled by physical processes. J. Plankton Res., 27: 61-70.

CARPENTER S. \& K. COTTINGHAM. 1997. Resilience and Restoration of Lakes. Conservation Ecology, [online] 1(1): 2. www.consecol.org/ vol1/iss 1/art2/

CASALLAS, G. J. E. \& G. GUNKEL. 2001. Algunos aspectos limnológicos de un lago altoandino: el lago San Pablo, Ecuador. Limnetica, 20(2): 215232.

CORDERO J., M. GUEVARA., E. MORALES \& C. LODEIROS. 2005. Efecto de metales pesados en el crecimiento de la microalga tropical Tetraselmis chuii (Prasinophyceae). Rev. Biol. Trop., 53(34): 325-330.

DASÍ, M. J., M. R. MIRACLE, E. CAMACHO, J. M. SORIA \& E. VICENTE. 1998. Summer phytoplankton assemblages across trophic gradients in hard-water reservoir. Hydrobiologia, 369/379: 27 43.

Del GIORGIO, P., A. VINOCUR, R. LOMBARDO \& H. G. TELL. 1991. Progressive changes in the structure and dynamics of the phytoplankton community along a pollution gradient in a lowland river-a multivariate approach. Hydrobiologia, 224: 129-154.

DOLBETH M., M. A. PARDAL, A. I. LILLEBLO, U. AZEITEIRO \& J. C. MARQUES. 2003. Short- 
and long-term effects of eutrophication on the secondary production of an intertidal macrobenthic community. Marine Biology, 10: 1133-1135.

EEAOC (Estación experimental Agroindustrial Obispo Colombres). 2008. Clima. Datos meteorológicos. Www.eeaoc.org.ar.

ENG-WILMOT, D. L., W. S. HITCHCOCK \&. D. F. MARTIN. 1977. Effect of temperature in proliferation of Gymnodinium breve and Gomphospheria aponina. Marine Biology, 41: 71-77.

FOGG, G. E., W. D. P. STEWART, P. FAY \& E. WALSBY. 1973. The blue-green algae. Academic Press. 437 pp.

FONTÚRBEL, F. 2004. Modelo operacional ambiental y aspectos sociales relevantes del proceso de eutrofización localizada en cuatro estaciones experimentales del lago Titikaka. Publicaciones Integrales, La Paz. 241 pp.

GUNKEL, G. 2003. Limnología de un lago Tropical de Alta Montaña, en Ecuador: Características de los sedimentos y tasa de sedimentación. Rev. Biol. Trop., 51: 381-390.

HENDEY, N. 1977. The species diversity inshore diatom communities and its use in assessing the degree of pollution insult on parts of the North Coast of Cornwall. Nova Hedwigia Beih., 54: 355378.

HÖFLE, M., H. HAAS \& K. DOMINIK. 1999. Seasonal dynamics of bacterioplankton community structure in a eutrophic lake as determined by 5S rRNA analysis. Appl. Environ. Microbiol., 65: 3164-3174.

HUSZAR, V. L. M., L. H. S. SILVA, P. DOMINGOS, M. MARINHO \& S. MELO. 1998. Phytoplankton species composition is more sensitive than OECD critera to the trophic status of three Brazilian tropical lakes. Hydrobiologia, 369/370: 59-71.

ISASMENDI, S. C., B. C. TRACANNA, F. H. VENDRAMINI, N. G. NAVARRO, M. A. BARRIONUEVO \& G. S. MEONI. 2007. Caracterización física y química de ríos de montaña (Tafí del ValleTucumán-Argentina). Limnetica, 26(1): 129-146.

LOCASCIO de MITROVICH, C., A. VILLAGRA de GAMUNDI, B. TRACANNA, C. SEELIGMANN \& C. BUTÍ. 1997. Situación actual de la problemática limnológica de los embalses de la provincia de Tucumán (Argentina). Lilloa, 39(1): 81-93.

LOEZ, C. 1995. Determinación de clorofila $a$. En: Ecosistemas de aguas continentales, metodologías pa- ra su estudio. Lopreto, E. C. \& Tell, G. (eds.): 263267. Vol. 1. Ediciones Sur, Argentina.

MARGALEF, R. 1969. El concepto de la polución en limnología y sus indicadores biológicos. Inv. $\mathrm{Hi}$ drol., suplem. Rev. Agua, 7: 103-133.

MARGALEF, R. 1983. Limnología. Editorial Omega. 1010 pp.

MOCHKOPSKY, L. 2002. Presa embalse La Angostura. Recursos hídricos del NOA. Provincia de Tucumán. Cuenca del Río Salí. Su aprovechamiento como aporte al desarrollo económico provincial y regional. Abstracts of the V Jornadas, Comité Argentino de presas Regional Norte, Tucumán, Argentina: 1-56.

NAUWERCK, A. 1994. A survey on water chemistry and plankton in high mountain lakes in northern Swedish Lapland. Hydrobiologia, 274: 91-100.

OLOFSSON, J. A. Jr. 1980. The role of microlayers in controlling phytoplankton productivity. In: $H y-$ pertrophic ecosystems J. Barica y L. R. Mur (eds.): 83-93. W. Junk, Publ. La Haya.

PRENDA, J., M. CLAVERO, F. BLANCO-GARRIDO, A. MENOR \& V. HERMOSO. 2006. Threats to the conservation of integrity in Iberian fluvial ecosystems. Limnetica, 25(1-2): 377-388.

PROSPERI, C. H. 2000. Competencia entre las cianoficeas y las pirroficeas en el embalse San Roque (Córdoba, Argentina). Abstracts of the Seminario Internacional "Identificación y control de algas en la producción de agua potable”, Santa Fe, Argentina: 60-64.

REYNOLDS, C. S. 1987. The response of phytoplankton communities to changing lake environments. Schweiz. Z. Hydrol., 49: 220-236.

REYNOLDS, C. S. 1998. What factors influence the species composition of phytoplankton in lakes of different trophic status. Hydrobiologia, 369/379: 11-26.

REYNOLDS, C. \& A. E. WALSBY. 1975. Water blooms. Biol. Rev. Cambridge Philos. Soc., 50: 437-481.

RODRÍGUEZ, M. I. y A. COSSAVELLA. 2000. Evaluación preliminar comparativa de la calidad del agua y sedimentos de embalses destinados al abastecimiento de agua potable del Gran Córdoba. Abstracts of the Seminario Internacional "Identificación y control de algas en la producción de agua potable”, Santa Fe, Argentina: 66-76.

RODRÍGUEZ, L. \& D. RIVERA. 1995. Efecto del cobre y cadmio en el crecimiento de Tetraselmis 
suecica (Kylin) Butcher y Dunaliella salina Teodoresco. Estud. Oceanol., 14: 61-74.

ROJO, C. 1998. Differential attributes of phytoplankton across the trophic gradient: a conceptual landscape with gaps. Hydrobiologia, 369/370: 1-9.

ROTT, E. 1998. Some aspects of the seasonal distribution of flagellates in mountain lakes. Hydrobiologia, 161: 159-170.

SEELIGMANN, C. \& B. TRACANNA. 1994. Limnología del embalse El Cadillal Tucumán Argentina. II: Estudio Cualitativo del fitoplancton. Cryptogamie, 15(1): 19-35.

SEELIGMANN, C. T. \& B. C. TRACANNA. 2003. Fitoplancton de un embalse de alta cota (TucumánArgentina). Bol. Soc. Argent. Bot., 38(Supl.): 165.

SEIP, K. L. \& C. S. REYNOLDS. 1995. Phytoplankton functional attributes along trophic gradient and season. Limnol. Oceonogr., 40(3): 589-587.

SHANNON, C. E. \& W. WEAVER. 1963. The Mathematical Theory of Communication. Univ. Illinois Press, Urbana, ILL, USA. 117 pp.

SHAPIRO, J. 1990. Current beliefs regarding dominance by blue-greens: the case for the importance of $\mathrm{CO}_{2}$ and $\mathrm{pH}$. Verh. Internat. Verein. Limnol., 24: 38-54.

SKULBERG, O. M., G. A. CODD \& W. W. CARMICHAEL. 1984. Toxic blue-green algal blooms in Europe: a growing problem. Ambio, 11: 244-247.

SLÁDECEK, V. 1973. Ergebnisse der Limnologie. Archiv für Hydrobiologie, Organ der Internationalen Vereinigung für theoretische und angewandte Limnologie. Beiheft 7. E. Schweizerbart'sche Verlagsbuchhandlung. Stuttgart. 218 pp.

TOPALIÁN, M. L., P. M. CASTAÑÉ, M. G ROVEDATTI \& A. SALIBIÁN. 1999. Principal component Analysis of Dissolved Heavy metals in water of the Reconquista River (Buenos Aires, Argentina) Bull. Environ. Contam. Toxicol., 63: 484-490.

TRACANNA, B. C., A. VILLAGRA de GAMUNDI, C. T. SEELIGMANN, C. LOCASCIO de MITROVICH \& E. DRAGO. 1992. Limnología del embalse El Cadillal, (Tucumán, Argentina).I: Va- riables morfométricas y físicoquímicas. Rev. Hydrobiol. Trop., 24: 269-286.

TRACANNA, B. C. \& C. T. SEELIGMANN. 1993. Limnología del embalse El Cadillal TucumánArgentina.III: Estudio cuantitativo del fitoplancton. Lilloa., 38: 65-74.

TRACANNA, B. C., V. MIRANDE \& C. SEELIGMANN. 1994. Variaciones del fitoplancton superficial del embalse Río Hondo (Tucumán-Santiago del Estero, Argentina), en relación a la actividad azucarera. Tankay, 1: 80-82.

TRACANNA, B. C., C. T. SEELIGMANN \& V. MIRANDE. 1996. Estudio comparativo de la comunidad fitoplanctónica de dos embalses del Noroeste Argentino. Rev. Asoc. Cienc. Nat. Litoral, Argentino, 27(1): 13-22.

TRACANNA, B. C., C. T. SEELIGMANN, V. MIRANDE, L. BENÍTEZ de PARRA, M. TORRES de PLAZA \& F. M. MOLINARI. 1999. Cambios espaciales y temporales del fitoplancton en el embalse Río Hondo (Argentina). Bol. Soc. Argent. Bot., 34: 101-105.

TRACANNA, B. C., S. N. MARTINEZ DE MARCO, M. J. AMOROSO, N. ROMERO, E. CHAILE \& A. MANGEAUD. 2006. Physical, chemical and biological variability in the Dr. C. Gelsi reservoir (NW Argentine): A temporal and spatial approach. Limnetica, 25(3): 787-808.

UTERMÖHL, H. 1958. Zur Vervollkommung der Quantitativen Phytoplankton Methodik. Mitt. Int. Ver. Limnol., 9: 1-38.

WESTERN, D. 2001. Human-modified ecosystems and future evolution. PNAS, 98: 5458-5465.

ZAVALA DE CHOKOFF, E. 2002. Inventario de presas. Provincia de Tucumán: Abstracts of the $V$ Jornadas, Comité Argentino de presas Regional Norte, Tucumán, Argentina: 1-13.

ZALOCAR DE DOMITROVIC, Y., V. M. ASSELBORN \& S. L. CASCO. 1998. Variaciones espaciales y temporales del fitoplancton en un lago subtropical de Argentina. Rev. Brasil. Biol., 58(3): 359-382. 